\title{
Análisis del proceso metodológico en programas de educación superior en modalidad virtual
}

\section{Analysis of the methodological process in higher education programs in virtual modality}

\author{
Paula Andrea Díaz-Guillen \\ Universidad de Manizales. Manizales, Colombia \\ pauladg@umanizales.edu.co \\ Yamilhet Andrade-Arango \\ Universidad de Manizales. Manizales, Colombia \\ yandrade@umanizales.edu.co \\ Ana María Hincapié-Zuleta \\ Universidad de Manizales. Manizales, Colombia \\ ahincapie@umanizales.edu.co \\ Adriana Patricia Uribe-Uran \\ Universidad Sergio Arboleda. Barranquilla Colombia \\ adriana.uribe@usa.edu.co
}

\begin{abstract}
Resumen
Este artículo identifica las características metodológicas de los programas virtuales de educación superior en Colombia, ante la carencia de lineamientos acerca de cómo implementar procesos de educación virtual a nivel de pregrado y posgrado en un país en vía de desarrollo. Se llevó a cabo una investigación con enfoque cualitativo en una muestra a propósito de 10 instituciones de educación superior en Colombia, con una oferta amplia de programas de pregrado y posgrado para la modalidad virtual. Los resultados de este estudio señalan que, en las instituciones de educación superior colombianas, se implementan modelos de enseñanza-aprendizaje que se modifican según las contingencias del contexto y las formas de concebir los diferentes roles que desempeñan los actores que participan en el proceso educativo. Particularmente, se encontró que dichas instituciones definen modelos educativos en los que la interacción asincrónica prevalece, evalúan mediante actividades parametrizadas en el campus virtual y forman sus docentes para mejorar sus competencias en el diseño de escenarios virtuales de aprendizaje. A nivel práctico, este estudio advierte que las características metodológicas de la educación virtual a nivel de pregrado y posgrado difieren de la educación presencial, por lo que las instituciones deben abordar ambas modalidades con estrategias pedagógicas diferentes.
\end{abstract}

Palabras clave: educación superior, modalidad virtual, proceso metodológico, calidad, inclusión digital.

\begin{abstract}
This article identifies the methodological characteristics of virtual higher education programs in Colombia, given the lack of guidelines on how to implement virtual education processes at the undergraduate and graduate levels in a developing country. A qualitative research was carried out in a sample of 10 higher education institutions in Colombia, with a wide range of undergraduate and graduate programs for the virtual modality. The results of this study indicate that Colombian Higher Education Institutions are implementing teaching-learning models that are modified according to the contingencies of the context and the ways of conceiving the different roles played by the actors participating in the educational process. In particular, it was found that these institutions define educational models in which asynchronous interaction prevails, evaluate through parameterized activities in the virtual campus,
\end{abstract}


and train their teachers to improve their competencies in the design of virtual learning scenarios. In practical terms, this study suggests that the methodological characteristics of virtual education at an undergraduate and graduate level differ from face-to-face education, so institutions should approach both modalities with different pedagogical strategies.

Keywords: higher education, virtual modality, methodological process, quality, digital inclusion.

\section{Introducción}

La formación en entornos virtuales es la respuesta de la masificación de la educación y la expansión de la sociedad del conocimiento en el mundo actual, el cual exige procesos tecno-pedagógicos alcanzables, sostenibles, dinámicos y flexibles. Este tipo de formación también es un modelo competitivo a escala regional, nacional e internacional que demanda la implementación de procesos administrativos, académicos y tecnológicos que permitan brindar educación de calidad desde cualquier lugar, espacio y tiempo. Como lo indican Melo y Díaz (2018), es necesario crear un escenario ideal que posibilite el desarrollo del proceso académico que, además de responder hacia unos requerimientos técnicos y procedimentales, también debe considerar variables diversas que contribuyan a una educación en entornos virtuales más efectiva.

Es así como las instituciones de educación superior han experimentado, a lo largo de la historia, transformaciones que exigen superar prácticas tradicionales desde los diferentes actores que intervienen en el proceso, para asumir el desafío actual que se presenta de generación, conservación, intercambio y transferencia de conocimientos (Basantes et al., 2018). Lo anterior exige cambios desde los diferentes procesos que acompañan la modalidad virtual, que permitan ofrecer educación a sectores diversos y plurales para dar respuesta a los intereses sociales, económicos y culturales de las naciones. De acuerdo con esta perspectiva, Arango et al. (2018) señalan que la educación superior en ambientes virtuales debe ser agente movilizador importante de innovación y transformación a nivel macro y micro del contexto social. Estas condiciones se deben reflejar en la descentralización de la educación, los objetos virtuales de aprendizaje (OVAS) y los entornos virtuales de aprendizaje (EVAS), mediante diseños ampliamente accesibles, tecno-pedagógicos y afectivos. Esto implica un sistema educativo que responda a la igualdad de oportunidades y que traspase las coordenadas geográficas, las condiciones económicas, la gestión pública, la política y la dinámica de las organizaciones.

Partiendo de ese escenario, en el que las instituciones de educación superior deben ser agentes de cambio, los posicionamientos clásicos centrados en el aula y la actividad del profesor que todavía se defienden con gran fuerza deben retornar a una enseñanza orientada hacia la actividad autónoma del estudiante, soportada en la interacción académica, lo asincrónico y las múltiples posibilidades de comunicación, elementos que deben ser evidentes tanto en la planeación como en los procesos de enseñanzaaprendizaje (Díaz, 2005). Esto advierte que es necesario promover un cambio de paradigma para poder generar propuestas educativas de calidad en lo virtual, las cuales

Análisis del proceso metodológico en programas de educación superior en modalidad virtual. Paula Andrea Díaz Guillen, Yamilhet Andrade Arango, Ana María Hincapié Zuleta y Adriana Patricia Uribe. 
permitan acortar distancias, optimizar el tiempo y ampliar los escenarios y los grupos de atención y actuación.

En este sentido, Escudero-Nahón (2017) define el desafío de la educación superior en modalidad virtual como la capacidad de conciliar las disposiciones pedagógicas y metodológicas que tradicionalmente han direccionado los procesos de formación desde lo curricular, lo administrativo y lo normativo de la educación presencial con la innovación y la transformación institucional de la modalidad virtual. En términos académicos, las instituciones de educación superior deben revisar los lineamientos y los procesos tecno-pedagógicos desde un referente distinto al habitual discurso pedagógico, el cual reconozca el diseño intencionado de objetos de aprendizaje y la creación de ambientes de aprendizaje con herramientas de interacción y comunicación que permitan "gestionar, administrar, distribuir, monitorear, evaluar y apoyar las diferentes actividades de formación" (Basantes, 2018, p.36). Por consiguiente, la educación virtual debe garantizar la inclusión de las Tecnologías de la Información y la Comunicación (TIC) en los procesos académicos de manera intencionada, y debe transformar los métodos pedagógicos para permitir que las personas diseñen su ambiente personal de aprendizaje, es decir, que este último responda hacia las diferencias y las necesidades individuales, como también hacia los intereses colectivos.

Es importante resaltar que la educación virtual ha penetrado con dificultad el sector educativo, por lo que algunas instituciones de educación superior aún muestran resistencia hacia la transformación de los métodos pedagógicos y hacia la adopción de modelos educativos novedosos que permitan ofrecer educación de calidad en el ciberespacio y con las características que esto implica. Por consiguiente, este articulo muestra entonces cómo 10 instituciones de educación superior en Colombia, que participaron de esta investigación, han adoptado procesos metodológicos diferentes respecto a la educación presencial que permiten incorporar las TIC en sus procesos de enseñanza-aprendizaje. Asimismo, se muestra en este artículo que estas Instituciones han acortado las barreras de tiempo y espacio, por lo que han sabido responder hacia las demandas del medio, las personas, el sistema educativo, la información y la infraestructura tecnológica.

\section{De la sociedad de la información (SI) a la sociedad del conocimiento: aplicación en el sector educativo}

En el campo educativo, la sociedad de la información y del conocimiento es una forma nueva de referirse al uso de dispositivos digitales, de tal modo de facilitar el aprendizaje y la utilización de modelos educativos que cumplan con los objetivos tecno-pedagógicos que en la actualidad se exigen (Pérez-Zuñiga et al., 2018). El ser humano está inmerso en una época influenciada por el progreso tecnológico que no se puede despreciar en el sector educativo. Entre la segunda mitad del siglo XVIII e inicios del siglo XIX, cuando la sociedad sustituye la economía basada en la agricultura, por una basada en la transformación de materias primas, nacen la sociedad urbana, la maqui-factura, el uso de capitales y la Sociedad Industrial; esta última reemplazada, hace ya varios años, por la SI (Sociedad Postindustrial), de la cual se conocen las primeras publicaciones realizadas por

Análisis del proceso metodológico en programas de educación superior en modalidad virtual. Paula Andrea Díaz Guillen, Yamilhet Andrade Arango, Ana María Hincapié Zuleta y Adriana Patricia Uribe. 
los sociólogos Alain Touraine y Daniel Bell, caracterizadas por señalar la importancia de la adquisición, la manipulación, la distribución y el intercambio de la información que cobran gran influencia en las dinámicas y las decisiones culturales, políticas, económicas y educativas. Sánchez (2016) enuncia que, desde finales del siglo XX, existen formas nuevas de orientación que rigen la sociedad: "sociedad de la información, sociedad del conocimiento y sociedad del aprendizaje", las cuales reflejan ideas, enfoques, tecnologías y sistemas que se entretejen para observar una compleja sociedad que contribuye con una mejora en la calidad de vida del ciudadano.

Particularmente, la SI surge con una serie de avances científicos, tecnológicos y unos intereses globalizadores como la diversidad cultural, la virtualidad, el desarrollo de nuevos escenarios de formación, la eliminación de fronteras, la revolución en el mercado laboral, entre otros. Esto hace que la información en la sociedad esté en el marco del poder como valor universal, y que los seres humanos sientan la necesidad de aprender a usarla para su desarrollo, toma de decisiones y evolución. La CEPAL (2018) señala que, en la actualidad, el ser humano afronta grandes desafíos, retos, oportunidades y describe que las TIC han pasado por 3 momentos importantes. La primera consiste en la comunicación entre las personas mediada por dispositivos; la segunda está impulsada principalmente por los nuevos medios disponibles para crear y divulgar información mediante tecnologías digitales; y la tercera corresponde la inteligencia artificial. Esto, según Salazar y Tobón (2018), representa profundos cambios, es decir, los flujos de información, las comunicaciones y los mecanismos de coordinación se están digitalizando en muchos sectores de la sociedad, lo que es un proceso que se traduce en la aparición progresiva de formas nuevas de organización social y productiva. Estas formas, obviamente, afectan el sector educativo.

Hablar de información, según Castells (2006), indica específicamente el atributo de una organización en la que la producción, la gestión, la utilización y la expansión de la información son elementos primordiales de productividad y poder en la sociedad de hoy. Esta noción tiene relación directa con la generación, el reconocimiento, el almacenamiento y la difusión de datos de forma ilimitada, algo posible por el desarrollo progresivo de las redes telemáticas, la influencia de las TIC, el auge del internet y el funcionamiento veloz que estos avances representan, lo que implica un cambio cultural e histórico en la sociedad. Estos cambios, obviamente, afectan también al sector educativo, especialmente a las instituciones de educación superior.

Para el Ministerio de Tecnologías de la Información y las Comunicaciones (MINTIC) (2015), la SI es una sociedad en la que las tecnologías que facilitan la creación, la distribución y la manipulación de la información juegan un papel importante en las actividades sociales, culturales y económicas. Por esto, la CEPAL (2018) reconoce la importancia del acceso universal a las TIC, así como de la infraestructura, incluida la conexión de banda ancha, respecto al cumplimiento de los objetivos de desarrollo sostenible y la realización de políticas públicas. En este marco de referencia, la sociedad del conocimiento no solo es la heredera de la SI, sino que va más allá y está principalmente centrada en la persona, es decir, en las relaciones humanas y no tanto en los productos y los servicios, sino en el conocimiento. Esto posibilita que más personas puedan crear, consultar, utilizar y compartir la información y el conocimiento.

Análisis del proceso metodológico en programas de educación superior en modalidad virtual. Paula Andrea Díaz Guillen, Yamilhet Andrade Arango, Ana María Hincapié Zuleta y Adriana Patricia Uribe. 
En este sentido, la SI se encarga de construir saber para el desarrollo (PérezZuñiga et al., 2018), por lo que ya no solo se habla del intercambio de información, sino de la interacción simbólica de carácter humano que permite transformar las formas de vida y de relación con conceptos nuevos de tiempo y espacio a través del acto comunicativo y educativo. Por esto, es que la SI permea completamente los procesos de enseñanza-aprendizaje que se llevan a cabo en el sector educativo.

Pedraja (2017) expone que la sociedad del conocimiento impone exigencias crecientes para las naciones, las organizaciones y las personas. Este nuevo paradigma de las TIC se organiza en torno a redes que posibilitan la prestación de servicios de forma más eficiente y con una mayor escalabilidad. Esto permite que los sujetos se conviertan en agentes activos de los diferentes ámbitos y sujetos partícipes que colaboran en la toma de decisiones frente a los desafíos y a las posibilidades actuales. Esto configura la morfología social nueva: simbologías, prácticas, valores, guías y modelos de comportamiento, uso de otros lenguajes y medios/instrumentos para transmitir el conocimiento, lo cual exige grandes esfuerzos de adaptación y caminos diferentes por la influencia que ejercen en la vida de todos las TIC.

Efectivamente, Sánchez (2016) explica que se observan cambios profundos en diferentes ámbitos: (1) en el político se producen cambios en la toma de decisiones que dependen cada vez más de una legitimación científica, lo que causa que los actores políticos dependan cada vez más de expertos y asesores; (2) en el educativo se observan cambios en las estructuras ocupacionales y se le otorga una creciente importancia a la educación. Un indicador es la transformación de las universidades de instituciones de elite a instituciones de educación superior masificada; (3) en el cultural, en el que a pesar de que apenas se debaten estos temas de la transición hacia la sociedad del conocimiento, sí se ha producido un intenso debate en torno a la globalización y al uso de Internet, lo cual indica un profundo cambio en los procesos culturales y en las interacciones sociales relacionadas con el uso de las nuevas TIC y los avances en las tecnologías de tráfico.

El paso de la SI hacia la sociedad del conocimiento implica elementos como:

- Instantaneidad: el espacio virtual nuevo ha hecho de la comunicación una acción inmediata, simultánea y rápida, sin dejar de mencionar los formatos de almacenamiento que evolucionan hacia una velocidad vertiginosa para favorecer esta acción con más capacidad. Los seres humanos tienen como escenario cotidiano diversidad de información y datos que posibilitan el intercambio de mensajes, reducción de las distancias espacio-temporales y generación de cambios estructurales en las organizaciones, para lo cual se requiere con urgencia nuevos enfoques, habilidades, conocimientos y escenarios de actuación (Abad \& Orón, 2015; González, 2019; Grande et al., 2016).

- Accesibilidad: aunque es una variable especialmente económica, dado que no solo depende de la riqueza de los recursos de infraestructura que tengan a disposición las diferentes regiones para los ciudadanos, sino de las posibilidades personales para acceder a un dispositivo (Abad \& Orón, 2015), la accesibilidad es un elemento que posibilita una

Análisis del proceso metodológico en programas de educación superior en modalidad virtual. Paula Andrea Díaz Guillen, Yamilhet Andrade Arango, Ana María Hincapié Zuleta y Adriana Patricia Uribe. 
estructura de acceso a la variedad y la cantidad de contenidos que se encuentran por todos lados, gracias al internet y a los diferentes recursos tecnológicos, informáticos y comunicativos, situación que se convierte en algo primordial para el escenario público contemporáneo y que exige enfrentar el reto de aprender a recibir, a aprovechar y a utilizar la información desde las necesidades y diferencias de cada contexto o persona, lectura, vidente u oyente, es decir, posibilitando el acceso a todos los usuarios que podrían ser potenciales (Soto \& Miró, 2016).

- Contenidos multimedia: el progreso de los medios informáticos de los últimos años coloca el ser humano en una posición cómoda para acceder a la información y para tener un conocimiento amplio de la cultura, la comunicación y la educación de diferentes regiones y contextos a la par del mundo entero, no solo a través de texto, sino por medio de nuevas formas de expresión como lo son los elementos o recursos multimedia: imagen fija, móvil, ambiental, digital y sonora, lo que hace que la información cobre vida. (Espinosa-Izquierdo et al., 2017). No se debe desconocer que, para esta sociedad, el texto es un elemento relevante, pero en el mundo de internet, los elementos que permiten la interactividad con todas sus posibles combinaciones tienen mucho valor porque permite desarrollar un material llamativo, formas nuevas de comunicación y otras posibilidades de enseñanza-aprendizaje para el usuario (Arango et al., 2018).

- Interactividad: el internet, como hito principal de esta sociedad, los medios de comunicación y las redes sociales se han convertido en escenarios de interacción social que no solo permiten que sus usuarios sean consumidores de información, sino generadores de datos y mensajes de toda índole desde la posibilidad de elegir, establecer el ritmo, cantidad y complejidad, además del tipo de relación que se quiere establecer con la información (Grande et al., 2016; Melo \& Díaz, 2018).

- Globalización de la información: el término de globalización responde a características como la universidad como componente esencial del proceso de creación de conocimientos en las juventudes y de las condiciones culturales e institucionales de carácter organizacional para la innovación de la sociedad. Además, admite una pluralidad de canales de transferencia y difusión de conocimiento y tecnología (Didrikson, 2000, citado por Rengifo, 2015). Como realidad actual y sustentada, también en la transformación de las telecomunicaciones y los avances técnicos y tecnológicos, la globalización ha hecho posible mostrar en las diferentes regiones del mundo, de manera casi instantánea, un modelo democrático de información que permite ahorrar recursos económicos (costos) y tener lugar en una realidad caracterizada por la desigualdad social. (Rodríguez, 2015). La revolución que ha generado esta época (el computador, los teléfonos celulares, el internet, la fibra óptica y sus facilidades) ha posibilitado el desarrollo humano a nivel universal.

- Eliminación de fronteras: el ser humano está inmerso en un escenario histórico y cultural que no funciona en el vacío, sino en un contexto local, regional, nacional e internacional, es decir, en un mundo global en la búsqueda de equidad social y oportunidades nuevas (Serrano, 2015). La eliminación de fronteras está proporcionando, a partir de la democratización de las TIC, la masificación de mercados en un ámbito

Análisis del proceso metodológico en programas de educación superior en modalidad virtual. Paula Andrea Díaz Guillen, Yamilhet Andrade Arango, Ana María Hincapié Zuleta y Adriana Patricia Uribe. 
competitivo, el libre comercio, el intercambio de información, los bienes y los servicios entre los diferentes países del mundo, el surgimiento de sociedades participativas y activas y mejoras en la comunicación, lo que permite eliminar fronteras entre los estados y fomentar el surgimiento de políticas públicas e inversión económica (Sánchez-Tórres et al., 2012).

Flórez-Romero et al. (2017) expresa que, hoy en día, se reconoce plenamente la existencia de una sociedad que, cada vez más en el centro de sus desarrollos, ubica la información y su tratamiento como núcleo vital de realizaciones; información que, por su naturaleza blanda e intangible, es capaz de producir diversos tipos de transformaciones, especialmente en innovaciones en todos los sectores. La sociedad del conocimiento se apoya en los dispositivos tecnológicos como estrategia especial para empoderar las nuevas maneras de hacer, vivir, sentir, incluso de pensar y realizar la cultura. Esto significa que la tecnología es el recurso clave que permite potenciar la gestión del conocimiento que sustenta los procesos de innovación. Para Arango et al. (2028) es un hecho que la cultura de las sociedades se ve influenciada por las culturas digitales, asunto que está modificando drásticamente las formas de transmitir y construir conocimiento, a lo que González (2018) se refiere como la buena utilización de las TIC desde aquellos aspectos vinculados con la producción (otras tecnologías maduras y nuevas), es decir, desde la concepción de los artefactos como una construcción social en cuyo desarrollo intervienen múltiples actores, intereses y valores.

Esto significa entonces que la digitalización y la facilidad de transmisión y comunicación son términos que caracterizan el origen de otro tipo de institución que se entreteje en la sociedad de la información y el conocimiento, pues debe formar ciudadanos para el siglo XXI (Flórez-Romero et al., 2017 ) y crear condiciones necesarias para impulsar la producción y el fomento de ambientes y objetos virtuales de aprendizaje, lo cual conduce a la construcción de un modelo educativo más amplio para los estudiantes, y que lo hace diferente por el uso intensivo de las TIC, el acceso, las relaciones entre los sujetos y el procesamiento de la información que genera la rápida evolución de la ciencia y la tecnología y sus aplicaciones así como un soporte estructural para gestionar estos entornos cambiantes (Ramírez-Montoya, 2015). Todo lo dicho implica que queda la posibilidad de crecimiento para seguir contribuyendo hacia la concreción de una sociedad basada en el conocimiento que permita superar los retos y aprovechar las potencialidades que el acceso abierto otorga, que genere inclusión digital y un papel activo de los diversos actores. En la medida en que esto sea posible, se puede hablar de una comunicación y una educación participativa, bidireccional y horizontal.

\section{La educación a distancia}

Dentro de las innumerables definiciones que se han dado sobre la educación a distancia, se encuentra claramente que las construcciones se acercan fuertemente desde la perspectiva de "distancia", lo cual indica la existencia de una brecha de tiempo y espacio que imposibilita la interacción directa entre los diferentes actores de un proceso académico. Catalán (2018) comprende este tipo de educación como:

Análisis del proceso metodológico en programas de educación superior en modalidad virtual. Paula Andrea Díaz Guillen, Yamilhet Andrade Arango, Ana María Hincapié Zuleta y Adriana Patricia Uribe. 
Una modalidad de estudio que pudo desarrollarse a través de los avances de las TIC; asimismo, a través de ella se ha buscado resolver problemas de carácter económico, social, de cobertura y acceso en torno a la educación, dada la posibilidad que descubre para desarrollar procesos de enseñanza-aprendizaje sin la necesaria coincidencia de espacio-tiempo entre los actores del proceso educativo-, lo que permite desdibujar algunas de las barreras que limitaban el acceso de ciertos sectores a la educación y en especial, a la universitaria. (p. 41)

Para lograr una aproximación mejor a la definición de esta modalidad educativa, es importante considerar que se trata de un proceso académico con características específicas, en el cual las tecnologías juegan un rol trascendental, permitiendo la generación de escenarios no convencionales para el desarrollo de los procesos, lo que, a su vez, invita a pensar de manera diferente los aspectos metodológicos y didácticos del proceso de enseñanza y aprendizaje.

Según el Ministerio de Educación Nacional de la República de Colombia -MEN(2015), se entiende la educación a distancia como una solución social a los problemas de cobertura y calidad que aquejaban un número elevado de personas, que deseaban beneficiarse de los avances pedagógicos, científicos y técnicos que habían alcanzado ciertas instituciones, pero que eran inaccesibles por la ubicación geográfica o bien por los elevados costos que implicaba un desplazamiento frecuente o definitivo a esas sedes. La definición de dicho ministerio no tenía en cuenta la posibilidad de que fuera una modalidad de educación al servicio de todos los seres humanos. Es por esto que la educación a distancia, en la actualidad, es una alternativa de formación que ofrece otras estrategias didácticas mediadas por las TIC y que, de alguna manera, obra según RamosAzcuy (2018) "como mecanismo moderador de la inclusión social” (p. 7).

\section{Educación virtual}

Entender la educación virtual como parte de la educación a distancia invita a reflexionar sobre la evolución misma que ha tenido la educación a través del uso de las TIC. TovarGálvez y Cárdenas-Puyo (2012) anotan que, según el auge y desarrollo de las nuevas tecnologías, se adoptan las generaciones o los niveles por los cuales ha pasado la educación a distancia. Por lo tanto, según esta concepción, el MEN resalta las tres generaciones ya mencionadas.

Al respecto, Tovar-Gálvez y Cárdenas-Puyo (2012) reafirman que, con la irrupción de las TIC, el modelo de educación abierta y a distancia tradicional se ha visto beneficiado por la aparición de medios nuevos de transmisión de información e interacción. En la actualidad, no se puede pensar este modelo, sin estar identificado como una condición indispensable para sus procesos y para el desarrollo de las nuevas tecnologías, lo cual se conjuga para permitir la creación de programas académicos en la modalidad virtual.

Según el MEN (2009), la educación virtual hace referencia que no es necesario que el cuerpo, el tiempo y el espacio se conjuguen para lograr un encuentro de diálogo o experiencia de aprendizaje. Desde esta perspectiva, la educación virtual es una acción que busca propiciar espacios de formación y se apoya en las TIC para instaurar una nueva forma de enseñar y de aprender. En consecuencia, la educación permite "un diálogo

Análisis del proceso metodológico en programas de educación superior en modalidad virtual. Paula Andrea Díaz Guillen, Yamilhet Andrade Arango, Ana María Hincapié Zuleta y Adriana Patricia Uribe. 
didáctico mediado por la tecnología entre los docentes de una institución y sus estudiantes ubicados en el ciberespacio, que aprenden de forma independiente y grupal" (Galindo, 2015, p. 26).

\section{Educación superior en modalidad virtual}

La educación superior, como agente de cambio en la sociedad y como herramienta en el mejoramiento fundamental dentro de las estructuras sociales, posee múltiples propósitos. En estos propósitos se encuentran la diversificación y la democratización de la educación, que dan respuestas oportunas y pertinentes en diferentes contextos. Además, muestra una capacidad de interacción dialógica según las necesidades sociales en sus distintos escenarios tanto globales como locales, en los que la educación se desenvuelve y se proyecta ocupando un espacio importante en dichos lugares.

A partir de estos escenarios, las universidades son percibidas como agentes de cambio y, además, como una plataforma potente con muchas posibilidades y grandes apuestas en el campo educativo, dando surgimiento a la necesidad de incrementar el espectro de actuación y de impacto. Es por esto que las TIC emergen como un horizonte lleno de oportunidades, teniendo en cuenta que la educación se puede considerar un bien escaso en países en vía de desarrollo como Colombia.

Se debe reconocer la importancia de la información y su gestión en la sociedad actual, entendiendo que la primera se encuentra en un crecimiento constante para dar respuesta a las necesidades del entorno. Especialmente, conceptos como "la sociedad de la información" y "la sociedad de conocimiento" desempeñan un papel importante en las dinámicas sociales y culturales, las cuales se ven influenciadas por las nuevas maneras de crear y trasmitir conocimiento mediado por las tecnologías y los recursos digitales (CEDUM, 2019). La inmediatez y la accesibilidad a la información renovaron las interacciones sociales y transformaron drásticamente las formas de hacer cultura. En este sentido, las TIC deben considerarse herramientas prácticas y enriquecedoras que apoyan el incremento de la trasmisión de conocimiento en los escenarios donde hacen presencia (CEDUM, 2019).

Es de anotar que la gestión del conocimiento ha crecido de distintas maneras para movilizar la innovación en los diversos sectores en los que influye el ser humano y, desde hace varias décadas, eventos relacionados con dicha gestión como la diversificación y la descentralización de los procesos de enseñanza-aprendizaje han experimentado avances importantes al mejorar la calidad de la educación virtual y a distancia, siendo las TIC las principales herramientas mediadoras de estas modalidades. En consecuencia, esto ha posibilitado una evolución constante en referentes tanto legales como teóricos, los cuales dan cuenta de los grandes avances en este campo.

Colombia, particularmente, se ha preparado para la implementación de la educación inclusiva a través de las TIC. Ejemplo de esto es la oferta educativa en las modalidades de estudio a distancia y virtual. Además, en Colombia se ha legislado apostándole al favorecimiento de la expansión, la calidad y el impacto de dicha educación en todos los espacios de inflexión a nivel nacional (urbano y rural). Según el Ministerio

Análisis del proceso metodológico en programas de educación superior en modalidad virtual. Paula Andrea Díaz Guillen, Yamilhet Andrade Arango, Ana María Hincapié Zuleta y Adriana Patricia Uribe. 
de Educación Nacional de Colombia (MEN) (2010), la educación a distancia y virtual se entienden como una solución social a los problemas de cobertura y calidad que aquejaban a un número elevado de personas en el país, quienes deseaban acceder a los avances pedagógicos, científicos y técnicos que han alcanzado ciertas instituciones, las cuales se han encontrado ubicadas, generalmente, en zonas urbanas centralizadas. Estas instituciones, según el MEN, cubrían solo las necesidades de las personas que residían en dichas zonas, lo cual generó brechas de desigualdad para las personas ubicadas geográficamente lejos de las instituciones y a quienes no podían solventar los elevados costos que implicaba trasladarse a instituciones universitarias de los centros urbanos en Colombia. En este sentido, el propósito de la educación a distancia y virtual consiste en crear alternativas y una aproximación para que la educación superior llegue a la fracción de la población que se encuentra en zonas de difícil acceso en Colombia, lo que otorga una posibilidad de establecer los programas académicos en otras plataformas y establece un espacio nuevo para el acto pedagógico.

En cuanto a los problemas conceptuales sobre la calidad de la educación a distancia en ambientes virtuales (EADAV), Amozurrutia y Moreno (2007) discuten la situación de la educación a distancia en el contexto estructural. Los autores abordan las diferentes apreciaciones de la calidad como estándar dentro de los procesos (escalas de valores), su aplicación a esta modalidad en ambientes virtuales, tomando en consideración las particularidades y el carácter innovador de este campo educativo, el uso y gestión de la información, y la implementación de herramientas y metodologías nuevas como bases de la formación.

Silvio (2009) enfoca su investigación en la importancia que se le debe dar a la formación desde los ambientes virtuales, destacando la necesidad de realizar permanentemente mejoras en las condiciones de este espacio y sus modelos pedagógicos por medio de la gestión y la innovación en estrategias efectivas de calidad. Asimismo, Silvio identifica las diferentes modalidades educativas (presencial, a distancia, virtual y no-virtual) y contextualiza el proceso de virtualización de la educación superior, entendiendo esto como las nuevas construcciones de actividades de aprendizaje, investigación, gestión, entre otros. El autor explica que la comunicación asincrónica se ubica en la modalidad de educación virtual y a distancia, es decir, que es una característica propia de esta modalidad que logra un proceso de interacción entre el docente y el estudiante que va más allá de coincidir en el tiempo y el espacio. Silvio concluye que la educación virtual se asocia con factores socioeducativos y tecnológicos que se vinculan para generar conocimiento. Parra (2009) reflexiona sobre la educación virtual a distancia desde una perspectiva teórica estructural-funcionalista, marxista y weberiana evaluando el impacto que esta tiene en la sociedad. Es decir, para Parra la virtualización de la enseñanza se destaca por su innovación y transformación del conocimiento, y genera una mayor igualdad tratándose de cobertura y acceso al sistema educativo superior con la ayuda de las TIC como base de enseñanza, aprendizaje y uso.

La educación virtual, el desarrollo de las tecnologías y el proceso de globalización, integrados como un movimiento mundial transversal al aprendizaje, producen que la virtualidad se convierta en un escenario nuevo generador de realidades, a medida que su crecimiento integra las dinámicas en ambientes virtuales y remotos a la cotidianidad

Análisis del proceso metodológico en programas de educación superior en modalidad virtual. Paula Andrea Díaz Guillen, Yamilhet Andrade Arango, Ana María Hincapié Zuleta y Adriana Patricia Uribe. 
académica, y nacen nuevas capacidades y competencias en el sujeto que se encuentra en vía de desarrollo. Peña (2010), desde una visión filosófica, plantea cómo la virtualidad es una tendencia en constante cambio, la cual está ligada al desarrollo de las TIC y al mercado actual, por lo que en su trabajo realiza una búsqueda del significado de lo virtual y hasta dónde puede llegar la educación superior en Colombia para transformar la educación virtual en el futuro.

Padilla-Beltrán (2016) habla de la educación virtual en Colombia contextualizando el uso de TIC a través de la historia, por lo que realiza un recorrido cronológico desde la educación presencial a la virtual, pasando por el $e$-learning y el $b$ learning. También, Padilla analiza la importancia de los modelos de formación en la educación superior y cómo se ha enriquecido con la implementación de las herramientas tecnológicas dentro y fuera del aula. Por otro lado, Areth-Estévez et al. (2015) exponen cómo en Colombia la educación virtual tiene una alta demanda en el mercado, pero también señalan lo elevadas que son las tasas de deserción de los estudiantes en esta modalidad, por lo que llegan a la conclusión de que es necesario profundizar en las necesidades y los factores que llevan al estudiante a abandonar la educación virtual, y a buscar soluciones efectivas y de impacto en la sociedad. Otras iniciativas conceptuales internacionales muestran una relación entre las habilidades del estudiante en el proceso de aprendizaje y las habilidades del docente en la creación de espacios sociales con apoyo de las TIC. Shea y Bidjerano (2009) destacan la importancia de fomentar el sentido de presencia social dentro de los espacios virtuales a través de la habilidad del docente/acompañamiento para impulsar los procesos de pensamiento crítico y el desarrollo de capacidades de los estudiantes dentro de los procesos cognitivos.

Los programas de educación superior, abordados desde los escenarios virtuales y el acompañamiento desde las TIC, fundamentan una propuesta educativa transfronteriza que apuesta a estrategias potenciadoras y transformadoras de país, que enmarcan esferas sociales y culturales, estableciéndose progresivamente como estándares sólidos, amplios y diversificados. Las universidades consideran esta propuesta no solo como estratégica, sino también como una iniciativa conectada cabalmente con una manera distinta de proyectar sentido y dar cumplimiento a los aspectos declarados misionales desde las esferas locales, regionales, nacionales e internacionales (CEDUM, 2019).

\section{La inclusión digital}

La tecnología está siendo usada por los individuos para mejorar su vida en muchos aspectos, por lo que la academia no se escapa de esta tendencia mundial, dado que, como bien sabe hacerlo, este sector ha aprovechado esta nueva alternativa para apostarle a una educación pensada en la modernidad y con anhelos de inclusión y descentralización que permita mayor cobertura sin abandonar la calidad. Castro-Rodríguez et al. (2019) expresan que "la integración de dispositivos digitales en la sociedad ha impactado en las políticas educativas de muchos países, llevándolos a considerar la competencia digital como aspecto clave en la enseñanza obligatoria y esencial para la inclusión social" (p.1). Esto conlleva a que, cuando se hable de inclusión digital, no solo se comprenda como la presencia de herramientas, dispositivos o mediaciones tecnológicas, sino como la posibilidad de brindar espacios de formación en los que se pueda participar, aprender y

Análisis del proceso metodológico en programas de educación superior en modalidad virtual. Paula Andrea Díaz Guillen, Yamilhet Andrade Arango, Ana María Hincapié Zuleta y Adriana Patricia Uribe. 
compartir en un escenario educativo con menos restricciones y con más plenitud. En este contexto, la educación tiene el desafío de construir sociedades inclusivas, lo que implica que las instituciones desde su autonomía formulen propuestas que garanticen una educación para todos, las cuales reconozcan las diferencias y las necesidades y, en efecto, potencien el desarrollo integral.

Castells (1999) afirma que el surgimiento de un sistema nuevo de comunicación electrónico, caracterizado por su alcance global y su integración de todos los medios de comunicación y su interactividad potencial, está cambiando la cultura humana. Esto ha hecho que las investigaciones y estudios en inclusión digital hayan ido incrementándose gradualmente, en la medida en que el tema pasó de ser una tendencia a ser una necesidad en muchos escenarios incluyendo el educativo, tanto a nivel nacional como internacional. Sin embargo, estos acercamientos se enfocan especialmente en los niveles de acceso desde posturas muy teóricas, lo que hace difícil operacionalizar los hallazgos por lo que se convoca a su reflexión inmediata desde las prácticas e instauración, dado que cada vez es más difícil concebir el mundo digital separado del mundo real. Dentro de esa revolución comandada por internet, la sociedad del conocimiento, las TIC y las redes sociales se modifican para siempre las maneras de concebir la educación y de comunicarse, estableciendo nuevos roles, vínculos y otras posibilidades de acceso a los diferentes niveles de educación, así como las formas de relacionarse entre sí (Bonavitta, 2018).

En este sentido, existe un interés generalizado de dotar las instituciones y los actores de competencias para poder aprovechar las TIC y lograr la idea que plantean Bradbrook y Fisher (2008) de las 5C: conectividad, capacidad, contenido, confianza y continuidad. Núñez et al. (2019) reafirman que la inclusión digital tiene ventajas en la educación superior, como el poder contar con la disposición de un amplio volumen de información como material de estudio y contenidos fácilmente accesibles y actualizables, además que brinda la posibilidad de deslocalizar el conocimiento, facilitar los procesos de autonomía y autoregulación de los estudiantes, y garantizar el desarrollo de competencias cognitivas y comunicacionales. Esto, según Berrío et al. (2017), "exige una constante desconstrucción tecnológica en las rutinas básicas de las personas" (p. 164). Por tales razones y partiendo de que los avances tecnológicos son rápidos y masivos, los docentes deben estar en capacidad de fomentar objetos de aprendizaje con estrategias que ayuden los estudiantes a generar, gestionar y evaluar los conocimientos, junto con el manejo de medios y recursos tecnológicos-didácticos y de organización del entorno de aprendizaje adecuados a las necesidades y expectativas de los estudiantes y de los contextos locales, nacionales y regionales.

El acceso inmediato a la información y el desarrollo tecnológico acortan la distancia de una sociedad del conocimiento como fuente de desarrollo para todos, jalonando cada día con más fuerza la transformación de los escenarios de educación y la emancipación de la dependencia geográfica. Por ello, la universalización del acceso a Internet se ha convertido en una prioridad de política para los países de América Latina y el Caribe (Galperín, 2017). Internet, como medio que permite la interacción, la comunicación y la organización social, comprendida específicamente como tecnoestructura de redes que hacen posible la resignificación de las experiencias, del

Análisis del proceso metodológico en programas de educación superior en modalidad virtual. Paula Andrea Díaz Guillen, Yamilhet Andrade Arango, Ana María Hincapié Zuleta y Adriana Patricia Uribe. 
conocimiento y de las prácticas de interacción humana (Cabrera, 2004), potencia el paradigma tecnológico actual para el desarrollo y alimenta el capital humano garantizado de manera gradual la disminución de la desigualdad social, sobre todo en países en vía de desarrollo. Sin embargo, no se puede desconocer que Buzato (2008) describe la inclusión digital como condición que marca los diferentes contextos por apropiaciones, pero también por conflictos relacionados con las TIC, dado que promueven otras estructuras de costo, modalidades nuevas de estudio, cambios en la forma de concebir el aprendizaje, la enseñanza y la evaluación.

Dussel (2014) considera "la inclusión digital como un proceso complejo y multidimensional que avanza en una topografía muy heterogénea, poblada de instituciones y culturas con su propia densidad" (p. 39). Esto justifica que no todas las instituciones estén preparadas para enfrentar retos nuevos y disponer de herramientas metodológicas que permitan el diseño de parámetros nuevos en la administración y la ejecución de programas y proyectos enmarcados en la virtualidad. Como bien lo manifiesta Galperín (2017), la formación online depende no solo del nivel de ingresos, sino de condiciones sociodemográficos que hacen evidente la separación de algunos grupos, los que están conectados a la revolución de las TIC y le apuestan a ello, los que aún tienen resistencia o no cuentan con las posibilidades bien sea económicas $\mathrm{u}$ organizacionales para implementarlas, o los que están excluidos de ellas, lo que comúnmente se denomina brecha digital y que, según Melo et al. (2018), representa la distancia que existe entre las personas, las empresas, las instituciones, las familias, las regiones, lo nacional, lo global y lo internacional, y las TIC e internet. Es importante resaltar la evolución que ha tenido la inclusión digital desde lo conceptual hasta el acceso, el uso y el nivel de apropiación tecnológica en un gran número de sujetos, escenarios políticos, educativos y sociales, y áreas geográficas. Sin embargo, habría que resaltar que, aun cuando las TIC son motores del desarrollo y representan el medio por el cual se accede y se genera conocimiento, faltan modelos competitivos que sirvan como referentes de transformación, movilización e innovación educativa (UNESCO, 2013).

En las instituciones de educación superior, la brecha digital no se da por la carencia de recursos o inversión, sino por la ausencia de habilidades tecnológicas de su personal docente, administrativo y organizacional con relación al nivel del desarrollo tecnológico, el acceso digital y la capacidad de actualización a la medida de los avances y de las nuevas propuestas. Es necesario comprender de manera consciente que la creación, la distribución y la manipulación de la información juegan un papel importante en las actividades sociales, culturales, económicas y académicas en el siglo presente. Según Núñez et al. (2019), esto exige un cambio curricular, actitudinal y de compromiso de los actores responsables del proceso como agentes catalizadores de las reformas y propuestas innovadoras en el aula presencial o virtual que, de una u otra forma, marcan el desarrollo integral del estudiante y el alcance de las competencias que le permitirán coexistir en lo que el mundo le exige hoy desde los diferentes contextos de actuación, pues el dominio y el uso de una tecnología o dispositivos digitales no solo dependen del sujeto, sino también de las oportunidades, apoyo y reconocimiento que su comunidad ya sea académica, laboral, cultural o social le brinde (Berrío et al., 2017). Es decir, no solo es cuestión de acceso a los recursos tecnológicos y de conectividad, sino que también es cuestión de poder ser garantes del uso de las TIC desde diálogos disciplinares, didácticos

Análisis del proceso metodológico en programas de educación superior en modalidad virtual. Paula Andrea Díaz Guillen, Yamilhet Andrade Arango, Ana María Hincapié Zuleta y Adriana Patricia Uribe. 
y pedagógicos que permitan movilizar las concepciones y las prácticas educativas en entornos virtuales.

En este sentido, las instituciones se constituyen en ejes fundamentales del proceso de inclusión, especialmente desde lo digital, pues desde sus escenarios de actuación y procesos misionales como la docencia, la investigación y la proyección social deben contribuir a la gestión de la información y su interpretación, usos y aplicabilidad, y a resolución de problemas reales en distintos contextos (Castro-Rodríguez et al., 2019). Es necesario enfrentar en la cotidianidad del aula y de sus prácticas de enseñanza-aprendizaje y de comunicación e interacción los desafíos que implican la construcción de escenarios nuevos que permitan crear alternativas pedagógicas y didácticas mediadas por las TIC que, sustentadas en los marcos y principios de la inclusión educativa, permitan la formación integral de todos los estudiantes. Esto significa tener las actitudes y aptitudes deseables para la comprensión y puesta en práctica de los retos pedagógicos que la inclusión digital propone en pro del desarrollo de la sociedad y la igualdad de oportunidades.

A partir de lo anterior y, en la búsqueda de alternativas para que la educación sea más humanizada y brindada con mayor equidad y calidad, se plantea una visión nueva de la educación en la actualidad, la inclusión, proceso en el que no se mira la educación orientada a un trato más igualitario en la medida de lo posible, sino una educación en la que la diversificación curricular y las metodologías que respeten diferentes ritmos de aprendizaje sean el privilegio de una enseñanza adaptada a todas las necesidades, tiempos, contextos y realidades. Como la tecnología es inherente a todos los procesos de desarrollo, es imperante que la inclusión digital sea una respuesta a la desigualdad social relacionada con el uso de las tecnologías y las posibilidades de acceder a la educación desde otras modalidades de estudio como las ofrecidas en entornos virtuales. Es necesario revisar las características, los alcances y las limitaciones de la brecha digital para crear políticas o lineamientos en todas las instituciones de educación superior donde se dé prioridad a la apropiación de las TIC, a la comprensión de las necesidades de las comunidades, a la generación de contenidos, conocimientos y capacidades de las personas para su uso (Rivoir, 2009).

En definitiva, es importante destacar que la brecha digital ha sido consecuencia de otras brechas de desarrollo y una de sus respuestas es la inclusión digital que puede leerse en primera instancia como desarrollo social, y que actúa como invitación para el planteamiento de planes, políticas y de procesos de atención a toda la población en general, es decir, como la posibilidad de alcanzar niveles de acceso, uso y beneficios a partir de las tecnologías. Esto da paso a la virtualización como nuevo paradigma educativo, lo que permite, según Rama (2014), cambios tecnológicos en los modelos societarios, con nuevas formas de gestionar y procesar la información, y concepciones diferentes sobre la construcción de competencias en el proceso educativo, permitiendo la atención desde las diferentes limitaciones y necesidades en un mismo lugar "el ciberespacio", en especial a partir de los desarrollos de las TIC que en su expansión muestran caminos y alternativas diversas para operar en el marco de la construcción diferenciada de ambientes de aprendizaje.

Análisis del proceso metodológico en programas de educación superior en modalidad virtual. Paula Andrea Díaz Guillen, Yamilhet Andrade Arango, Ana María Hincapié Zuleta y Adriana Patricia Uribe. 


\section{Educación a distancia y virtual en el contexto colombiano}

Las reflexiones suscitadas por la educación a distancia y virtual son muchas. Por esto, en esta sección, se abordan algunas reflexiones provocadas a lo largo de su proceso histórico, que si bien este tipo de educación no podrá ser un tema muy novedoso por los numerosos escritos e investigaciones que existen alrededor del tema en la literatura (Gros-Salvat, 2011), sigue siendo un tema utópico y relevante, máxime si se parte de que el fenómeno que estructura la realidad presente se conoce como TIC, con miras a la universalidad de la educación permanente.

Ahora bien, hablar de universalidad exige transformar el sistema convencional de educación formal e informal para poder responder hacia las múltiples demandas educativas de la sociedad y de la cultura progresivamente diversificada. En la actualidad, la educación ofrecida en esta modalidad a distancia-virtual ha mostrado un crecimiento considerable gracias al incremento de la conectividad y la disminución de los costos en el servicio de internet (Estévez et al., 2015). En el contexto colombiano, el Consejo Nacional de Acreditación menciona que la educación a distancia y virtual es una estrategia metodológica para ampliar las oportunidades de acceso a la educación superior y, en consecuencia, facilita el ingreso a esta metodología educativa de un mayor número de estudiantes (MEN, 2010). Es posible que esto sea discutible, pues las Instituciones de Educación Superior ven en este tipo de modalidad oportunidades para expandir su operación.

En este sentido, el sistema educativo colombiano ha crecido como en los demás países, gracias al auge del internet en los últimos años y a su actuación como factor de desarrollo social, económico y educativo. Según el MEN (2010), la educación a distancia $\mathrm{y}$ virtual es un modelo alternativo y complementario que responde a las necesidades de una numerosa población, puesto que elimina la concepción de que el ser humano coincida en toda actividad en las coordenadas de espacio y tiempo. Las tecnologías nuevas han permitido la existencia de formas nuevas de comunicación, la globalización de la información y la construcción de conocimiento. Estas transformaciones exigen a la humanidad una mirada diferente de la educación desde los términos de apertura, flexibilidad e innovación (Díaz, 2005).

La transición del siglo XX al siglo XXI se caracterizó por el descubrimiento de internet, las telecomunicaciones y la enseñanza asistida por computadora, lo que dio paso a la educación centrada en el estudiante y a su interactividad con el material de estudio y los tutores/profesores que, según Roquet-García (2006), se trataba de una etapa caracterizada por el auge de la comunicación vertical y bidireccional en forma instantánea (comunicación sincrónica) y diferida (comunicación asincrónica). Asimismo, se da una comunicación e interacción entre los estudiantes (comunicación horizontal y trabajo colaborativo). Es a esto a lo que hoy llamamos educación en línea. Es importante resaltar que la comunicación instantánea fue un medio acogido primero por la industria, las empresas públicas, la banca, entre otros, para luego instalarse en el escenario pedagógico, hecho que puede ser justificado dentro de la cultura conservadora que ha tenido la comunidad educativa a lo largo de la historia frente a los cambios que la sociedad brinda con respecto a los procesos metodológicos de formación.

Análisis del proceso metodológico en programas de educación superior en modalidad virtual. Paula Andrea Díaz Guillen, Yamilhet Andrade Arango, Ana María Hincapié Zuleta y Adriana Patricia Uribe. 
Visto de otra manera, la influencia de los medios de comunicación en la educación se puede resumir en tres generaciones de enseñanza que Garrison (1985) identifica como correspondencia, telecomunicación y telemática, y una cuarta que Taylor (1999) define como enseñanza vía internet, cuya aparición se debe a la rápida influencia de los medios de comunicación en la educación, los avances tecnológicos y la capacidad de los estudiantes de trabajar en red. Desde su emergencia, la educación a distancia según el MEN (2010) ha pasado por tres generaciones:

Primera generación, se caracteriza por la utilización de una sola tecnología y la poca comunicación. El estudiante realiza su trabajo en solitario. (2) Segunda generación, introdujo otras tecnologías y una posibilidad de interacción. Además del texto impreso, el estudiante recibe casetes de audio o video, programas radiales y cuenta con el apoyo de un tutor. (3) Tercera generación, de la educación a distancia se caracteriza por la utilización de tecnologías más sofisticadas y por la interacción directa entre el profesor del curso y sus alumnos. A esta última generación de la Educación a Distancia se la denomina "Educación Virtual". Desde esta perspectiva, entonces, la educación virtual es una modalidad de la Educación a Distancia y corresponde a la generación más avanzada de la misma. (p. 37)

Este breve recorrido hace evidente cómo las TIC en el tiempo han transformado entornos sociales, económicos, culturales y educativos a partir de su uso generalizado y la digitalización de la información, que de alguna manera ha suplido necesidades y limitaciones de acceso a las personas proporcionando posibilidades sin precedentes. La creación de estas tecnologías y su inclusión en la sociedad han llevado a cambios en la actividad diaria de la humanidad, expresado por Carreras (2008) de la siguiente manera:

Desde hace unos años nuestra sociedad se ha ido cambiando de forma vertiginosa, cambiando aspectos del sector productivo, de las formas de relación e incluso las potencialidades de comunicación. Una de las razones de esta transformación tan importante, que afecta cualquier esfera de la vida cotidiana, ha sido la incorporación de las tecnologías de la información y comunicación (TIC) en nuestras vidas. Estas tecnologías han modificado todos los ámbitos laborales y científicos de forma radical, incluso un sector como el cultural que se ha interrumpido más tardíamente. (p.19)

Estos cambios, si bien han sido producto de manifestaciones históricas, la utilización de los diferentes medios de comunicación no se pueden ubicar en épocas o años diferenciales, pues en la actualidad y según la influencia política, social, cultural, económica, entre otros, a nivel regional y local, hay quienes imparten la educación a distancia y virtual haciendo uso de los diferentes recursos que a lo largo de la historia han ido evolucionando (el computador, redes de transmisión de datos, plataformas educativas, correo postal, correo electrónico, texto escrito, CD, DVD, USB, entre otros), para garantizar la comunicación oral, escrita y audiovisual. Desde una mirada integral, la educación a distancia y virtual ha permitido eliminar barreras de espacio, tiempo y

Análisis del proceso metodológico en programas de educación superior en modalidad virtual. Paula Andrea Díaz Guillen, Yamilhet Andrade Arango, Ana María Hincapié Zuleta y Adriana Patricia Uribe. 
distancia, e ir transcendiendo en la maduración de sociedades con índices de buena calidad de formación.

La educación a distancia se puede entender entonces como "un método o sistema educativo de formación independiente, mediada por diversas tecnologías" (JucaMaldonado, 2016, p. 1) que, según González (2018), "permitió el acceso a la formación académica de aquellas personas que tenían esa necesidad y que, por motivos de distancia, tiempo y económicos, se veían imposibilitadas de acceder a la formación presencial" ( $p$. 59) y, finalmente, "se basa en un diálogo didáctico mediado entre docentes de una institución y los estudiantes que, ubicados en espacio diferente al de aquellos, aprenden de forma independiente o grupal" (García-Aretio, 2014, p. 2).

Por tanto, las TIC se han convertido en el ambiente cotidiano de la educación, lo que contempla la transformación de una comunicación dominada por la escritura y el teléfono, a otra dominada por el internet, dando paso a nuevas lógicas de comunicación entre docentes y estudiantes. Esta metodología convierte el rol del estudiante pasivo en uno activo constructor de su aprendizaje. Así mismo, el rol del docente o tutor ya no se concibe como un experto en la temática transmisor de conocimientos, sino como un guía, un acompañante del estudiante en la construcción del andamiaje del conocimiento, es decir, que construye puentes entre lo que sabe y las estrategias de aprendizaje mediadas por las TIC para ir motivando no sólo al estudiante, sino la discusión y el proceso de formación.

En síntesis, hoy, la educación a distancia y virtual se ha ganado un escenario protagónico en los sistemas de capacitación y/o formación, lo que da cuenta de las excelentes posibilidades para garantizar una educación permanente. El avance de las TIC desde las cartillas o guías didácticas a la televisión y la radio y, en los 90, a la incorporación de redes satelitales, el correo electrónico y el uso masivo del internet han permitido pensar en una educación a distancia y virtual, definida por Salazar et al. (2013) como:

Un conjunto de relaciones pedagógicas entre estudiantes, docentes e institución, basadas o apoyadas en el uso de tecnologías para el desarrollo sistémico de procesos formativos de calidad. Esta metodología educativa promueve la inclusión y la movilidad social, fundamentada en el aprendizaje autónomo y la autogestión, que utiliza pedagógica y didácticamente diversas metodologías, mediaciones y estrategias, en las que incorpora el uso de medios y tecnologías disponibles y accesibles, para la provisión y certificación del servicio educativo de la formación integral, al que puede acceder el estudiante sin barreras geográficas, de tiempo, edad, género, raza, etnia, credo religioso, condiciones políticas, sociales, culturales, de aprendizaje, o nacionalidad. (p. 102)

Lo anterior corrobora que la educación a distancia y virtual surge como respuesta a facilitar el acceso a aquellas personas que no podían participar en la formación presencial, y permite que el estudiante y el profesor estén físicamente separados durante el proceso de aprendizaje y mantengan la comunicación en una variedad de formas. Por lo tanto, se reconoce como una experiencia que se puede hacer fuera de una institución

Análisis del proceso metodológico en programas de educación superior en modalidad virtual. Paula Andrea Díaz Guillen, Yamilhet Andrade Arango, Ana María Hincapié Zuleta y Adriana Patricia Uribe. 
académica, en casa o en un lugar de trabajo. El aprendizaje es abierto, flexible, negociado $\mathrm{y}$ adaptado a las necesidades de cada persona.

\section{La calidad en la educación}

Hablar de calidad en cualquier escenario implica una valoración juiciosa, rigurosa y exigente de los procesos que se estén cualificando. Particularmente, hablar de calidad en la educación superior supone el desafío de buscar la excelencia. La calidad en las instituciones educativas de educación superior se consolida como el resultado de múltiples procesos llevados a cabo por distintos actores que tienen un objetivo general y que se desarrollan en el marco de altos estándares que se convierten en el común denominador. La educación virtual tiene un mayor reto al intentar demostrar a través de procesos curriculares, pedagógicos, metodológicos y de evaluación desarrollados de manera intencionada, que puede seguir contribuyendo con la universalización y la democratización del conocimiento con altos estándares de calidad (Arango et al., 2018).

Una educación de buena calidad, independientemente de la modalidad, debe proporcionar a los estudiantes las capacidades necesarias para que sean económicamente productivos, para desarrollar una calidad de vida sostenible, para contribuir a la construcción de una sociedad democrática y para mejorar el bienestar individual y colectivo del entorno en el que se encuentre; configurándose incluso como uno de los diecisiete Objetivos del Desarrollo Sostenible de las Naciones Unidas, que se basa en la firme convicción de que la educación es uno de los motores más poderosos y probados para garantizar el desarrollo sostenible, así como proporcionar acceso igualitario a formación técnica asequible y eliminar las disparidades de género e ingresos, además de lograr el acceso universal a educación superior de calidad (UNESCO, 2017).

La calidad es un concepto se ha venido transformando, teniendo sus primeras definiciones en los procesos industriales y organizacionales, llegando después al ámbito educativo. Según Peña-Seminario y Aponte-González (2018), en la sociedad industrial se tenía una perspectiva interna que se enfocaba en el producto y su permanecía por largos periodos de tiempo de manera exitosa en el mercado. Ya en lo educativo, dicha calidad se medía por resultados y logros, más que por el proceso metodológico y de planeación, por el criterio de conformidad de los usuarios del servicio de formación y por el valor de la venta en comparación con el costo de funcionamiento y puesta en marcha de los programas.

Más adelante en la sociedad de la información, el concepto de calidad se traslada a una perspectiva más externa, que involucra los conceptos de eficacia y competitividad, con una visión de la educación como servicio, obteniendo su valor por el nivel de satisfacción del cliente. Ya en la sociedad del conocimiento, con una perspectiva globalizada de la excelencia como criterio que define los procesos educativos, se busca que la satisfacción que antes se localizaba en el usuario del servicio, se traslade a todos los actores involucrados en el proceso de formación generando un compromiso por todos que sea coherente con el objetivo de formar las nuevas generaciones.

Análisis del proceso metodológico en programas de educación superior en modalidad virtual. Paula Andrea Díaz Guillen, Yamilhet Andrade Arango, Ana María Hincapié Zuleta y Adriana Patricia Uribe. 
Las instituciones y los programas de educación superior, según GonzálezGonzález et al. (2011), a través del análisis de Peña-Seminario y Aponte-González (2018), deben estar enfocados desde cuatro perspectivas: desde el ser en función de sus resultados y su eficacia; desde el quehacer, en función de su estructura funcional y eficiencia; desde el deber ser en función de su misión y pertinencia y, finalmente, desde el querer ser, en función de su proyecto de desarrollo en términos de la trascendencia.

La obtención de programas de calidad en la modalidad virtual involucra los esfuerzos de quienes desarrollan los grandes procesos misionales de la educación superior. Así pues, los docentes, los investigadores y quienes administran los programas desde la gestión se ven retados a comprender todas las aristas que emergen al consolidar, diseminar y administrar este tipo de programas, generando una visión holística que abarque todas las perspectivas. En el caso de la educación virtual, por un lado, están el rol de estudiantes y docentes, la metodología para la enseñanza-aprendizaje, la investigación, la estructura curricular que involucre los objetivos de aprendizaje, así como el diseño de los créditos académicos y, finalmente, la evaluación. Por otro lado, están los desarrollos tecnológicos puestos al servicio de la educación, la interacción y el intercambio de conocimiento (Arango et al., 2018).

La oferta de programas virtuales va en ascenso a nivel mundial, lo que implica para las instituciones de educación superior que los desarrollan tener criterios claros, ordenados y sistemáticos de evaluación de los procesos, que permitan medir, ya sea en el marco de criterios internos de la institución, o dentro de los procesos de acreditación de calidad, con los estándares dispuestos por las políticas de cada país para otorgar dichos reconocimientos, si los conocimientos que se generan en los estudiantes tienen algún impacto en la sociedad al poner en función de la misma. Los expertos en procesos de calidad dicen que algo puede mejorar solamente si puede ser medido, pero especialmente ser medido solamente si existen estándares contra los que se pueda comparar el proceso o el producto que se está generando (Cantón, 2000).

En Colombia, la calidad en los programas de educación superior es regulada por el sistema de aseguramiento de la calidad (SACES) y la comisión nacional intersectorial de aseguramiento de la calidad en la educación superior (CONACES), instituciones que se relacionan de forma directa con el MEN, que tienen como objetivo principal el evaluar las instituciones de educación superior, dando cumplimiento a los lineamientos basados en las categorías de: estudiantes, profesores, procesos académicos, entre otros, mediante procesos de autoevaluación (Ministerio de Educación Nacional, 2016) desde los Decretos 1075 de 2015 y 1330 de 2019, los cuales hacen referencia a las características propias de programas en modalidad virtual que se deben cumplir (Tinoco-Giraldo \& Giraldo, 2019).

Por último, y no menos importante, en un proceso de formación virtual de calidad, está la relación que se establezca entre los estudiantes y las plataformas educativas, las cuales se convierten en el vehículo a través del cual el estudiante encuentra los materiales, las actividades, los cronogramas y los contenidos dispuestos por el docente de manera intencionada y planeada e interactúa con ellos. Esta experiencia es fundamental para mejorar la disposición del estudiante frente al aprendizaje. Un programa de calidad no

Análisis del proceso metodológico en programas de educación superior en modalidad virtual. Paula Andrea Díaz Guillen, Yamilhet Andrade Arango, Ana María Hincapié Zuleta y Adriana Patricia Uribe. 
puede permitir el uso indiscriminado de las TIC, el exceso de recursos y las actividades puede desenfocar al estudiante del proceso de aprendizaje (Fainholc, 2004).

\section{Metodología}

La metodología empleada en esta investigación tuvo un enfoque cualitativo. De acuerdo con esto, se realizaron entrevistas en las que participaron rectores, docentes y coordinadores de 160 programas (ver Tabla 1), en los niveles de formación de pregrado y posgrado de 10 instituciones de educación superior de Colombia seleccionadas mediante un muestreo a propósito. El criterio de selección de las instituciones consistió en que tuvieran programas de educación virtual a nivel de pregrado y posgrado. Las entrevistas realizadas se enfocaron en obtener información sobre las categorías siguientes: (1) proceso de enseñanza-aprendizaje; (2) proceso de evaluación; (3) perfil del estudiante; (4) perfil docente-tutor; (5) créditos académicos; y (6) investigación. Estas categorías posibilitaron comprender los procesos metodológicos que en educación virtual implementaron las instituciones de educación que participaron en el estudio.

Tabla 1

Relación programas de pregrado y posgrados en modalidad virtual entrevistados

\begin{tabular}{|c|c|c|c|}
\hline Institución & Posgrado & Pregrado & Total \\
\hline 1 & 22 & 17 & 39 \\
\hline 2 & 2 & & 2 \\
\hline 3 & 12 & 9 & 21 \\
\hline 4 & 8 & 6 & 14 \\
\hline 5 & 10 & 12 & 22 \\
\hline 6 & 10 & 1 & 11 \\
\hline 7 & 7 & 4 & 11 \\
\hline 8 & 3 & 5 & 8 \\
\hline 9 & 12 & 9 & 21 \\
\hline 10 & 6 & 5 & 11 \\
\hline Total & $\mathbf{9 2}$ & $\mathbf{5 1}$ & $\mathbf{1 6 0}$ \\
\hline
\end{tabular}

Fuente: elaboración propia con base en la información del MEN, 2020.

El análisis de los datos obtenidos de las entrevistas realizadas se efectuó mediante un proceso de codificación por patrones, de acuerdo con las categorías que se definieron previamente. La investigación tuvo dos etapas. En la primera etapa se ejecutaron las siguientes actividades:

Análisis del proceso metodológico en programas de educación superior en modalidad virtual. Paula Andrea Díaz Guillen, Yamilhet Andrade Arango, Ana María Hincapié Zuleta y Adriana Patricia Uribe. 
- Mediante la primera actividad se identificaron las características de la modalidad virtual en instituciones de educación superior en Colombia, lo que posibilitó la comprensión de experiencias educativas virtuales y teorías educativo-culturales. Esta fase implicó identificar instituciones colombianas de educación superior con programas de pregrado y posgrado en modalidad virtual. Posteriormente, se identificaron elementos comunes de los fundamentos de teóricos de la educación virtual, lo cual direccionó el planteamiento de las categorías y la estructuración de la entrevista y las preguntas respectivas.

- En una segunda actividad, a partir de la base de datos de instituciones de educación superior con programas de pregrado y posgrado orientados en modalidad virtual, se eligieron las instituciones mediante un muestreo a propósito. Se buscaron los datos de contacto de directores de programa y encargados de los centros de apoyo de los procesos de virtualidad. Posteriormente, se hizo un primer contacto vía telefónica con el fin de socializar el objetivo del proyecto y solicitar la participación en la investigación, así como las posibilidades de agenda para llevar a cabo las entrevistas.

- En una tercera actividad, con la respuesta de aceptación de las universidades y siendo una investigación financiada mediante convocatoria interna de investigación Universidad de Manizales, se redactó una carta formal de invitación para colaborar en esta investigación, firmada por el representante legal de la Universidad, el señor rector, las cuales fueron enviadas vía correo electrónico y entregadas de manera presencial en la fecha y hora de la entrevista.

- En una cuarta actividad se realizaron entrevistas a partir de desplazamiento a las instituciones, lo cual permitió en su mayoría no solo hacer la entrevista, sino conocer de manera cercana sus modelos virtuales, recursos multimedia, plataforma, campus, procesos y actores, entre otros.

En la segunda etapa se ejecutaron las siguientes actividades:

- En primer lugar, se sistematizó la información de las entrevistas llevadas a cabo y se realizó el análisis de los datos cualitativos obtenidos a partir de las categorías anteriormente definidas.

- En segundo lugar, se realizó una revisión de literatura que permitió comprender y confrontar los elementos encontrados en las entrevistas, con el fin de entretejer ideas, posturas, cuestionamientos y configurar los resultados.

- En tercer lugar, se realizó la codificación de las respuestas obtenidas mediante el proceso de entrevista.

Análisis del proceso metodológico en programas de educación superior en modalidad virtual. Paula Andrea Díaz Guillen, Yamilhet Andrade Arango, Ana María Hincapié Zuleta y Adriana Patricia Uribe. 


\section{Análisis de los resultados por categorías}

Este análisis permitió la creación de conceptos y la generación de discurso teórico a partir del análisis de datos que promueve la calidad de la educación y la inclusión digital en escenarios de educación superior en la modalidad virtual.

\subsection{Enseñanza-aprendizaje}

El modelo de enseñanza-aprendizaje en los programas ofertados en modalidad virtual se ha caracterizado, según las universidades entrevistadas, en trabajar referentes tales como selección de un modelo pedagógico que permita la articulación en estrategias de ambientes virtuales. Este modelo responde a las necesidades que se van dando en un ecosistema de aprendizaje y requiere de un trabajo de articulación con los objetivos de aprendizaje esperados en los programas académicos, la cultura evaluativa y el diseño de un sistema de seguimiento que facilite la integración y la articulación de los resultados de aprendizaje esperados, los cuales se han adaptado a modelos metodológicos que contemplan estrategias tecnológicas como mecanismos que dan prioridad en el desarrollo de sus actividades académicas. Así pues, en esta modalidad de enseñanza, las TIC se integran como recursos de apoyo a los procesos de enseñanza/aprendizaje.

Sin embargo, cabe resaltar que pocos programas consideran que sus estrategias de enseñanza-aprendizaje requieren de unos encuentros presenciales para garantizar un mejor seguimiento en estrategias de los resultados que esperan para que el estudiante pueda alcanzar; mientras que la mayoría de programas se apoyan de sus plataformas educativas para que de forma sincrónica y asincrónica se cumpla una metodología que responda a las necesidades de aprendizaje.

En este sentido, se identificó que el proceso de enseñanza-aprendizaje va ligado al paradigma que apunta a la responsabilidad y la autonomía de los estudiantes, por lo que es necesario hablar que la educación en modalidad virtual responde a esquemas de autoformación, destacando que en todos los programas se cuenta con el acompañamiento de profesores que están en todo el proceso de aprendizaje. No obstante, es el estudiante quien, mediante su compromiso, desarrolla cada una de las estrategias propuestas para que aprenda acorde con las características de su nivel de formación.

Asimismo, se identifica que algunos de los programas presentan estrategias diferentes para el desarrollo de un modelo de enseñanza-aprendizaje, el cual consiste en un trabajo mediante estudios de caso y el modelo de aula invertida. En estas estrategias los entrevistados manifiestan un mayor grado de participación de los estudiantes, lo cual se refleja en una mejor interacción y apropiación de los temas desarrollados en clase. Para la mayoría de los entrevistados, las estrategias de aprendizaje que implementan las Instituciones de Educación Superior están basadas en actividades que se dan mediante el trabajo autónomo y colaborativo identificado en modelos tradicionales de aprendizaje.

Análisis del proceso metodológico en programas de educación superior en modalidad virtual. Paula Andrea Díaz Guillen, Yamilhet Andrade Arango, Ana María Hincapié Zuleta y Adriana Patricia Uribe. 
A continuación, se presenta la evidencia textual cualitativa de la síntesis que se acaba de presentar:

"Se tiene un documento que básicamente es el modelo de educación virtual, este se maneja en todas las facultades como base y es el que rige cuando se implementa algún programa virtual. Este cuenta con tres partes, pedagogía, tecnología y comunicación, en el centro de esto está el estudiante como un sujeto autónomo en el que se puede identificar como esta persona aprende y colocamos estos tres ejes al servicio de esa persona para que pueda aprender". (E1)

"Se cuenta con un modelo integral que busca desde lo profesional hasta lo humano, se fundamente en un modelo praxeológico que aborda cuatro grandes aristas, ver, juzgar, actuar y la devolución creativa. Su aplicación se verifica a través de la plataforma que la Universidad utiliza donde el aprendizaje, las actividades e incluso las rubricas están bajo este diseño y asistido todo por TIC". (E2)

"Constructivismo, Conectivismo. se puede evidenciar con el comportamiento de los estudiantes, los sucesos en el aprendizaje y la recepción del conocimiento". (E3)

"El modelo UNADISTA se basa en la fusión del aprendizaje colaborativo, aprendizaje autónomo y aprendizaje colaborativo. Se verifica mediante la construcción de los cursos, los cuales deben contener actividades orientadas hacia el desarrollo los tres tipos de aprendizaje señalados. En efecto, la evaluación se orienta hacia estos tres tipos de aprendizaje". (E4)

"Se tiene un enfoque centrado en el estudiante, en que este mejore su proceso académico, se busca incrementar el trabajo colaborativo". (E5)

"Escenarios para el aprendizaje es la estrategia más importante, donde de manera interactiva de vincula al estudiante con el proceso de formación”. (E6)

"Se da un acompañamiento continuo de todos los tutores, todo pensado en el estudiante que está en el centro del ecosistema con las particularidades y problemas que tiene este estudiante". (E7)

"Al mando de todo esto está el docente de curso correspondiente, manejamos el bienestar virtual a través del centro de psicología virtual por medio de canales, plataformas y softwares que les permite llevar el proceso". (E8)

"Se cuenta con el bienestar universitario mirando desde todos los aspectos, uno desde el componente tecnológico donde garantizamos a los estudiantes con los tutores y profesores en nuestra plataforma una respuesta oportuna a los estudiantes virtuales; también contamos con actividad deportiva virtual, guitarra, comunicación y eficacia, estilos de vida saludable, y otros cursos todos en la plataforma". (E9)

Análisis del proceso metodológico en programas de educación superior en modalidad virtual. Paula Andrea Díaz Guillen, Yamilhet Andrade Arango, Ana María Hincapié Zuleta y Adriana Patricia Uribe. 
"Gestión de estudiantes, la cual incluye servicios como bienestar universitario, consultoría académica y movilidad”. (E10)

\subsection{Proceso de evaluación}

Según las universidades entrevistadas, los procesos de evaluación se hacen a través del diseño de diferentes estrategias que permiten evidenciar interacciones, apropiaciones conceptuales, procedimentales y de investigación, productos, procesos y habilidades. Prevalece en los programas online la evaluación formativa que se da a lo largo del proceso de aprendizaje y que permite ir validando el alcance de las competencias para hacer planes de retroalimentación, acompañamiento y mejora. En todos los casos, las Instituciones de Educación Superior privilegian la evaluación acumulativa en la que se validan o aprueban y desaprueban cuantitativa y cualitativamente las asignaturas o módulos según los planes académicos de cada programa (se evalúa el proceso y el resultado). Prevalece en las respuestas que las universidades cuentan con la planeación de:

- Actividades individuales en las que el estudiante reconoce sus propios procesos cognitivos, conocimientos, actitudes y comprueba los logros del aprendizaje como también su aplicación en los diferentes contextos.

- Actividades colaborativas en las que los estudiantes interactúan en grupo en torno a una actividad, situación o postulado, lo que propicia que no estén solos en su proceso de aprendizaje, sino que aprenda con el otro, se logren metas comunes y se desarrollen habilidades tanto individuales como colaborativas.

Es de resaltar que el 9\% de los programas presenta un modelo de evaluación que conjuga el aula invertida, la cual refiere la discusión de un tema específico, la cooperación y el desarrollo de guías. Algunas instituciones trabajan con estudios reales que exigen, en primera instancia, el esfuerzo individual para luego ser resuelto en equipoconstruccionismo. Pocas instituciones evalúan a través de rúbricas, es decir, a través de criterios en un marco de autoevaluación, reflexión y revisión por pares. Prevalece, según la codificación de las respuestas entregadas en las entrevistas realizadas, que las Instituciones de Educación Superior evalúan a través de cuestionarios y otras actividades como foros, mapas mentales, ensayos, resúmenes, proyectos, exposiciones, videos, posters, talleres, estudios de casos, entre otras posibilidades que plantean los docentes teniendo en cuenta lo disciplinar, los intereses curriculares, las apuestas metodológicas y de tiempo en relación al crédito. En cuanto al escenario de presentación pocas Instituciones de Educación Superior, en sus programas online, exigen que las evaluaciones y laboratorios se hagan de manera presencial. Prevalece en las respuestas que los programas online trabajan desde el ciberespacio a través de plataformas elearning.

Lo anterior indica que la evaluación es un proceso constitutivo de los procesos de enseñanza-aprendizaje y que todo sistema de educación virtual tiene contemplada, al interior de sus acciones formativas, la evaluación para identificar los avances en el desarrollo del ser humano en el proceso y en el contexto a través de la relación interactiva entre el estudiante el contenido y la actividad, desde sus vivencias y experiencias en

Análisis del proceso metodológico en programas de educación superior en modalidad virtual. Paula Andrea Díaz Guillen, Yamilhet Andrade Arango, Ana María Hincapié Zuleta y Adriana Patricia Uribe. 
concordancia con el principio del aprendizaje autónomo y colaborativo, lo cual permite determinar si los resultados de aprendizaje, habilidades, objetivos o competencias planteados en los programas y evidenciados en las asignaturas o módulos fueron logrados. Esto advierte que la evaluación es pensada para identificar aciertos y dificultades existentes en el proceso educativo. Emitir juicios acerca de la progresión y la calidad del aprendizaje implica un proceso sistémico que va desde la planificación minuciosa del proceso hasta la vigilancia y el acompañamiento de la evolución de los estudiantes en el proceso de formación desde el ciberespacio.

A continuación, se presenta la evidencia textual cualitativa de la síntesis que se acaba de presentar:

"Los tipos de evaluaciones son diferentes, hay unas que se diseñan desde el proceso de producción del material educativo sean lúdicas, cuestionarios, etc. y estas no se repiten, hay preguntas abiertas, ensayos, resúmenes, en general el docente crea sus propias evaluaciones y las sube a plataforma, con las respectivas orientaciones" (E2)

"Definidos previamente por el área de operaciones, los docentes no pueden modificar las fechas de presentación de las actividades, los estudiantes deben enviar justificación cuando ellos no cumplan con las actividades en los tiempos establecidos. los módulos se dividen en RA: Refuerzo autónomo donde el estudiante presenta evaluaciones parametrizadas en plataforma; e INVESTIGACIÓN: donde el docente presta más apoyo en la calificación de entregas y trabajos en grupo.” (E4)

"Contamos con una guía del aspecto temático la cual tiene una estructura de cómo se hace, un listado de recursos digitales, una guía de evaluaciones específicas individuales y colaborativas, donde tenemos diferentes formas de evaluar para que el docente también elija desde allí, o si se le ocurre otra forma también." (E6)

"Evaluamos nuestro contenido y nuestro proceso de educación virtual en la plataforma con estudiantes, y los estudiantes se reúnen con la mediadora de educación virtual, con el coordinador, decano y se hace una evaluación de profesores y otra en la plataforma que se encarga de evaluar como fue el profesor, acompañamiento virtual y como están distribuidos los contenidos, al final se hace una reunión con los estudiantes para ver cómo les pareció todo y así poder hacer las mejoras respectivas en el diseño del material de estudio y las evaluaciones individuales y colaborativas." (E7)

"Preguntas con base en cartillas y lecturas fundamentales, simuladores de gestión." (E9)

"El currículo es puesto a consideración de los directores de programa con base en los núcleos problémicos que aborda éste último. Se realizan focus group con docentes de los programas para evaluar la idoneidad del currículo y construir las evaluaciones que deberán presentar los estudiantes en grupo y de manera

Análisis del proceso metodológico en programas de educación superior en modalidad virtual. Paula Andrea Díaz Guillen, Yamilhet Andrade Arango, Ana María Hincapié Zuleta y Adriana Patricia Uribe. 
autónoma. Adicionalmente, se revisa el desempeño de los estudiantes y las tendencias externas de las temáticas relacionadas con los cursos ofertados." (E10)

\subsection{Perfil del estudiante}

Para los rectores, profesores y coordinadores de los 160 programas de pregrado y posgrados, el estudiante es el centro para lograr su aprendizaje. Sin embargo, con el fin de garantizar un adecuado inicio respecto a este modelo de aprendizaje mediado por las TIC, se utilizan estrategias que van desde la alfabetización digital, programas de nivelación en el manejo de la plataforma educativa y rutas virtuales. Se resalta que el hecho de estar realizando un proceso de formación en modalidad virtual no indica que el estudiante cuente con las competencias digitales para comprender este modelo de formación.

En este sentido, se destaca que un número importante de estudiantes que recurren a la formación en modalidad virtual lo hace por la flexibilidad en el manejo tiempoespacio, lo que facilita poder trabajar o atender otras ocupaciones sin preocuparse de tener que hacer un desplazamiento o cumplimiento de un horario determinado para el desarrollo de sus actividades.

A continuación, se presenta la evidencia textual cualitativa de la síntesis que se acaba de presentar:

"Para la alfabetización contamos con varios programas, de igual manera el curso de inducción en el plan de formación tiene que ver con la alfabetizar a estos estudiantes que necesitan desarrollar ciertas habilidades ofimáticas”. (E1)

"A los estudiantes se les hace un curso y se les enseña cómo funciona y como se estudia en la virtualidad". (E2)

"Todos los estudiantes que ingresan a la modalidad virtual deben hacer el módulo de inducción el cual contiene información del programa, la universidad, el modelo metodológico, los recursos y actividades, los medios de interacción, instancias académico-administrativas, el cual está activo durante todo su proceso de formación”. (E3)

"Se tiene un curso que se llama manejo de la plataforma, ese curso está estipulado para 5 días con todas las actividades, de cómo se comunica con el profesor, con el tutor y como realiza todas sus actividades allí”. (E4)

"La inducción tiene un componente importante del uso de todas las plataformas y de cómo se comportan dentro de la institución de manera digital”. (E5)

"Se cuenta con dos formas, la primera es un recurso audiocontenido donde se explican todas las bondades de la virtualidad, de igual manera se le apuesta a que las herramientas sean muy fáciles de utilizar, también se tiene un recurso con tutor que cuenta como es la experiencia de educarse virtualmente”. (E9)

Análisis del proceso metodológico en programas de educación superior en modalidad virtual. Paula Andrea Díaz Guillen, Yamilhet Andrade Arango, Ana María Hincapié Zuleta y Adriana Patricia Uribe. 
“Rutas virtuales y formación en alfabetización digital”. (E10)

\subsection{Perfil docente-tutor}

La investigación refiere que la educación en modalidad virtual exige opciones pedagógicas diferentes a las convencionales y, además, maneras distintas de proyectar la universidad a través del uso de distintos instrumentos, herramientas, medios y dispositivos llenos de contenido y de sentido los que, en términos generales, se constituyen en las mediaciones que dotan de valor los componentes del acto educativo, permitiendo así la distribución de la información y la interacción, el proceso de evaluación y seguimiento. Además, confiere la responsabilidad del proceso al estudiante mediante las orientaciones que encuentra en el aula virtual o el campus para avanzar en la construcción y la reconstrucción de sus aprendizajes. Esto significa que el docentetutor es el agente facilitador de la capacidad autoreguladora del estudiante, a causa de que propicia el entorno y los materiales educativos y didácticos necesarios para alcanzar las metas de formación.

Lo anterior se soporta en los conocimientos y habilidades que los docentes-tutores deben tener tales como: (1) disciplinares, pues el docente-tutor es quien, según la mayoría de los entrevistados, tiene a cargo el diseño/construcción de contenidos y material de estudio, además de la orientación, el seguimiento y la evaluación del proceso de aprendizaje; en alguno de los programas se cuenta con profesores expertos en el área que construyen los contenidos y pueden o no orientarlos posteriormente, es decir, hay más un compromiso de producción del material de estudio; y solo el 18\% de los programas cuenta con comités académicos, vicerrectorías académicas o líderes de currículum que tienen la responsabilidad del desarrollo curricular y microcurrícular, planes de estudio, contenidos y guías didácticas. Como soporte a estos procesos, los docentes-tutores, en la mayoría de los programas, cuentan con el apoyo de algunos actores como asesores pedagógicos, personas formadas en educación, comunicadores audiovisuales y multimediales, diseñadores gráficos, diseñadores web y visuales, ingenieros, administradores de sistemas y expertos disciplinares que acompañan al docente-tutor en el proceso y los direcciona en diferentes aspectos propios de la modalidad, responden inquietudes, capacitan, publican contenido y material en las plataformas, y garantizan que no haya similitud de la información a través de las revisiones que hacen y los resultados arrojados por herramientas como Turnitin y Unicheck, dado que pocas programas cuentan con políticas previas, lineamientos, reglamentos o estatutos de propiedad intelectual. Finalmente, en algunos programas se exige al docente-tutor certificación en formación de herramientas telemáticas y digitales, proyecto académico pedagógico y competencias básicas y específicas en educación a distancia y virtual.

(2) Técnicos y académico-administrativos, pues en algunos programas, el docente-tutor es el vínculo personal-mediador entre el estudiante y las diferentes instancias administrativas, el proceso académico, el docente, los contenidos y los recursos tecnológicos; pocos programan cuenta con tutores técnicos, plataformas de apoyo y tutor virtual; y algunos programas cuentan con coordinadores académicos y directores de programas que apoyan esta labor; para algunas instituciones, el vínculo para cada una de

Análisis del proceso metodológico en programas de educación superior en modalidad virtual. Paula Andrea Díaz Guillen, Yamilhet Andrade Arango, Ana María Hincapié Zuleta y Adriana Patricia Uribe. 
las instancias presentadas están en el campus virtual, allí se tienen acceso directo a actores del proceso académico y del proceso administrativo. (3) Pedagógicos y aspectos propios de la modalidad, pues las universidades, en su mayoría, ofrecen capacitación, planes de actualización y formación profesoral, diplomados o cursos en lineamientos de educación virtual y el uso de recursos tecno-pedagógicos que permitan que los docentes-tutores estén preparados para orientar en dicha modalidad e innovar en el aula con estrategias o herramientas. Este es un proceso de formación permanente que se ofrece al inicio de cada semestre y durante el mismo. Es de resaltar que en algunos de los programas se le exige al cuerpo académico de la institución la certificación que garantiza la apropiación de competencias para el desempeño de actividades académicas, pedagógicas, tecnológicas, investigativas y de gestión académica en el marco de la modalidad de educación abierta y a distancia en escenarios telemáticos y virtuales. En general, los programas diseñan las formas de contacto e interacción entre los profesores (tutores, monitores) y los estudiantes para cada uno de los momentos de trabajo y evaluaciones propuestas en la plataforma o el campus a través de consejería virtual, encuentros sincrónicos y asincrónicos, actividades de foro, chat y correos.

"Cuentan con múltiples tutores, y plataformas de apoyo". (E1)

"Los profesores y tutores son los encargados del seguimiento y evaluación del proceso de aprendizaje". (E2)

"Los docentes o expertos temáticos son los encargados del diseño del material de estudio". (E3)

"Un experto temático y el asesor pedagógico quien es el encargado de instruir al profesor en cómo debe construir el diseño pedagógico, para que al final se construya el curso virtual”. (E4)

"Contamos con líderes de currículo, los cuales se encargan de los asesores pedagógicos institucionales, los cuales son una psicóloga, una comunicadora y una licenciada que son quienes se encargan de crear o diseñar el contenido". (E5)

"Los encargados de la producción de contenidos son los docentes con la ayuda de pedagogos, personas formadas en educación, comunicadores, comunicadores audiovisuales y multimediales, diseñadores gráficos, diseñadores web, y diseñadores visuales". (E6)

"El tutor es el encargado del desarrollo del componente académico les escribe permanentemente a los estudiantes". (E7)

"El docente planea y prepara el material de estudio, y ya las formas de contacto son la plataforma y la consejería virtual en ciertos aspectos". (E8)

"Se implementa el teletrabajo, es decir que los docentes no permanecen en la universidad, pero deben tener una disponibilidad total para los estudiantes y actividades académicas, la mayoría de esto a través de la tecnología”. (E9)

Análisis del proceso metodológico en programas de educación superior en modalidad virtual. Paula Andrea Díaz Guillen, Yamilhet Andrade Arango, Ana María Hincapié Zuleta y Adriana Patricia Uribe. 
"Contamos con profesores de diversas partes del mundo, ya que si podemos formar estudiantes de cualquier parte del mundo también podemos enseñar sin importar en donde nos encontremos". (E10)

\subsection{Créditos académicos}

En Colombia, para todos los programas en cualquier nivel de formación como pregrado, posgrados u otro, se establece mediante el decreto 1330 de 2019 que un crédito académico es igual a 48 horas, los cuales se representan en tiempo de contacto directo de interacción sea con el docente o en una plataforma educativa mediante un encuentro sincrónico o asincrónico y la relación de trabajo independiente. En este sentido, se encontró que, de los 160 programas, por sus diferentes niveles de formación, de los cuales el 58\% eran de posgrados y el $42 \%$ de pregrado, todos manejan la relación del crédito académico en diferentes proporciones.

En la mayoría de programas de posgrado prevalece la relación $1 / 3$, es decir que, por cada hora de contacto, hay 3 horas de trabajo independiente lo que significa que por cada crédito académico se ofrece a los estudiantes 12 horas de encuentro o contacto directo y 36 horas de trabajo independiente; en los programas de pregrado un porcentaje importante tienen una relación $1 / 2$, es decir que, por un crédito académico, hay 16 horas de interacción directa y 32 de trabajo independiente.

En comparación al tiempo de un programa en modalidad presencial, la representación con base al crédito académico es similar. No obstante, un bajo número de programas de posgrados presentaron una relación diferente en el tiempo directo en el que se expresa un tiempo de 6 horas de trabajo directo con el estudiante y 42 horas de trabajo independiente. Así mismo para el caso de pregrado, presentaron una relación menor en tiempo de contacto directo con el estudiante de 8 horas y 40 horas de trabajo independiente.

Con base a lo expresado por los entrevistados, lo importante más allá de la relación del crédito es su organización desde un proceso metodológico y pedagógico intencionado que permita que el estudiante interactué con lo propuesto en el aula virtual o campus para que alcance los resultados de aprendizaje deseados.

A continuación, se presenta la evidencia textual cualitativa de la síntesis que se acaba de presentar:

"Se hace en la misma proporción de los programas presenciales, 16 horas de actividades de interacción con lo que planea el docente y la plataforma y todo esto se garantiza a través de una tabla de producciones la cual contiene múltiples videos, actividades y recursos educativo con la cual se busca regular y estandarizar la relación del crédito y el tiempo". (E1)

"Los cursos son de 178 créditos al igual que en la modalidad presencial, 16 semanas, y 16 horas de contacto con el estudiante y 32 horas de trabajo autónomo

Análisis del proceso metodológico en programas de educación superior en modalidad virtual. Paula Andrea Díaz Guillen, Yamilhet Andrade Arango, Ana María Hincapié Zuleta y Adriana Patricia Uribe. 
y colaborativo, y cuentan con el material y la programación de cada asignatura entregado con anterioridad". (E2)

"Se maneja de manera integral, no hay algo estipulado como tal, se tienen unos mínimos de encuentros sincrónicos y de ahí para allá lo que el profesor planee para trabajar con los estudiantes y cumplir con los propósitos planeados”. (E3)

"Se establecen módulos cada uno de 8 semanas, con un total de 5 periodos académicos al año, cada uno liderado por un solo profesor, cada módulo cuenta con un núcleo integrado por 4 objetos con una intensidad de 8 créditos, para obtener un total de 40 créditos al año aspirando a culminar la carrera en un lapso de 4 años. Se trabaja una relación $1 / 3$ para posgrado y $1 / 2$ para pregrado. Se tienen unos lineamientos pedagógicos y metodológicos que se deben cumplir con el fin de garantizar escenarios pedagógicos y de enseñanza-aprendizaje planificados por parte del docente o tutor". (E4)

"La especialización tiene un promedio de 20 créditos, la maestría un promedio de 50 créditos y el pregrado tiene un promedio de 150 créditos. Se trabaja las 48 horas del crédito en la misma relación que presencial, haciendo distinción que en posgrado son por cada hora de trabajo de interacción con el campus 3 de actividades fuera del aula, de consulta, de investigación, indagación”. (E5)

"En el aula se encuentra todo el detalle de dicha información, ya que si bien se maneja una relación para planear lo importante es que esté alineado a lo que quiere alcanzar el programa en la formación de sus estudiantes". (E6)

"Nuestra Universidad trabaja para pregrados una relación 1/5 y para posgrado una relación 1/7, en esta relación las horas de contacto directo equivalen a la programación de videoconferencias con el docente donde se explica temática y se hacen ejercicios de interacción, no es obligatoria la asistencia, de hecho, los exámenes de programan los fines de semana para garantizar que los estudiantes puedan visualizar las clases grabadas posteriormente. En la mayoría de posgrados las actividades quedan abiertas al inicio y finalización década módulo”. (E7)

"Se trabaja con una relación 1/7- donde la única interacción real con el docente que se ofrece es un chat voluntario con los estudiantes los sábados, de resto todo está en la nube en la plataforma”. (E8)

"La institución da utilidad al 20\% que el MEN permite de presencialidad en programas virtuales, tenemos 16 horas de contacto, pero esas 16 horas se dividen en el tiempo de revisión de material en plataforma, lectura guiada, asesorías sincrónicas y asincrónicas, videoclases, recursos multimedia y el restante correspondiente al $20 \%$ ya mencionado para presentar exámenes en la sede de la Universidad de manera presencial". (E9)

"Lo determinado por el MEN, 48 horas la unidad del crédito y 16 y 36 horas". (E10)

Análisis del proceso metodológico en programas de educación superior en modalidad virtual. Paula Andrea Díaz Guillen, Yamilhet Andrade Arango, Ana María Hincapié Zuleta y Adriana Patricia Uribe. 


\subsection{La investigación}

El término investigación es uno de los criterios de calidad evaluados por el MEN en Colombia y debe ser transversal en los programas de formación profesional y posgradual ofrecidos en las diferentes modalidades como función sustantiva. Sin embargo, es un tema que genera aún ausencias, preocupaciones y pocas claridades en lo virtual. La mayoría de los entrevistados responde que vinculan el estudiante a este proceso igual que en presencial, a través de la investigación formativa, semilleros y participación en grupos de investigación. Estos son realizados en aulas virtuales por medio de encuentros sincrónicos o asincrónicos, es decir, lo que cambia es la mediación a través de las TIC. Algunos de los entrevistados invitan los estudiantes a participar en proyectos de investigación identificados previamente por los líderes de programas o coordinadores de líneas de investigación. Pocos programas vinculan los estudiantes a procesos de investigación desde que inician hasta que finalizan su proceso de formación, por lo que es un proceso obligatorio y trasversal. Muy pocos entrevistados expresaron que cada programa es responsable de plantear sus estrategias con sus estudiantes correspondientes y también pocos entrevistados consideran que la Institución de Educación Superior crea programas y proyectos para la generación de semilleros de investigación virtuales y desarrollo de práctica investigativa virtual.

Con ello se reconoce también desde los datos generales arrojados en las entrevistas, que hablar de la investigación no sólo supone reconocer los impactos que los medios tienen sobre estos procesos, sino que se hace necesario materializar el modo como la educación en ambientes virtuales orienta e impacta como práctica social y como construcción de lo digital desde la formación investigativa con producción científica y académica articulada con las necesidades de los gobiernos y las exigencias del contexto.

A continuación, se presenta la evidencia textual cualitativa de la síntesis que se acaba de presentar:

"Al momento de vincular a los estudiantes a la investigación lo hacemos por medio de semilleros como si fuera una universidad presencial, estos son realizados en aulas virtuales por medio de encuentros sincrónicos". (E3)

"Se hace de la misma forma que en la modalidad presencial". (E4)

"Para vincular a los estudiantes virtuales a la investigación se implementa que el estudiante sepa que la universidad va con él a todos lados, es decir que cuenta con todos los servicios de esta en todo momento". (E5)

"Esto lo maneja cada programa con sus estudiantes correspondientes". (E6).

"Igual que en presencial, a través de semilleros y participación en grupos de investigación, lo que cambia es la mediación". (E7)

Análisis del proceso metodológico en programas de educación superior en modalidad virtual. Paula Andrea Díaz Guillen, Yamilhet Andrade Arango, Ana María Hincapié Zuleta y Adriana Patricia Uribe. 
"Creación de programas y proyectos para la generación de semilleros de investigación virtuales y desarrollo de práctica investigativa virtual”. (E8)

"Se invitan los estudiantes a participar en proyectos de investigación identificados previamente por los líderes programas, los cuales están definidos en las líneas de investigación". (E9)

\section{Discusión}

El análisis de las respuestas dadas por los diferentes expertos de las instituciones de educación superior colombianas, que participaron de esta investigación, permitió observar también cómo existen elementos comunes y consistentes en la interpretación de aspectos esenciales de la educación virtual de los 160 programas de las diferentes universidades, en lo que corresponde a su naturaleza específica, la necesidad de formación de estudiantes y docentes, el uso de infraestructuras y métricas que determinan el uso y la accesibilidad de los recurso educativos y herramientas, el interés por responder a un proceso metodológico diferente a lo presencial, así como el mejoramiento continuo a partir de las evaluaciones permanentes que hacen de los proceso técnicos, tecnológicos y pedagógicos, y las dudas y sugerencias que se suministran al final de las asignaturas/módulos o cursos. Dejó ver también que hay una cultura de confianza en el estudiante en los procesos de aprendizaje y autoformación, dado que solo el $7 \%$ requieren la presencia de los estudiantes en el escenario físico de la universidad para presentar las evaluaciones y el 93\% trabaja con modelos de interacción académica desde la sincronía, asincronía y la interacción con las plataformas y el campus. Existe una mayor preocupación por afinar la investigación y articularla más desde la realidad, lo dinámico e intencional, el trabajo a partir de convenios, redes o comunidades académicas.

La investigación dejó ver cómo la educación superior tanto pública como privada en escenarios virtuales responde a tres de las dimensiones que plantea la Organización de las Naciones Unidas en el Plan Nacional Decenal de Educación 2016-2026: (1) accesibilidad entendida como la capacidad de eliminar fronteras desde las facilidades; (2) promoción de la inclusión y generación de múltiples posibilidades para quienes históricamente han estado excluidos del escenario educativo; y (3) aceptabilidad que exige la constitución de lineamientos para ofrecer procesos de educativos con calidad y adaptabilidad, lo cual permite la permanencia en el sistema educativo de más personas y garantiza la educación para la actualización permanente y la continuidad en su prestación.

Los resultados de la investigación señalan que las instituciones emplean métodos nuevos de enseñanza y técnicas nuevas de trabajo en las que no es necesario que el tiempo, el cuerpo y el espacio se conjuguen para lograr diálogos y experiencias de aprendizaje. Como bien lo manifiesta Otero de Suárez et al. (2016), en la actualidad se cuenta con una gama de alternativas que permiten que los estudiantes interactúen con diferentes formas de representación del conocimiento que posibilitan la abstracción de un objeto o situación de estudio. Su empleo facilita la participación, la distribución de roles y estimula el uso de diferentes tipos de medios audiovisuales participativos. Las instituciones de educación superior en esta modalidad están respondiendo de acuerdo con las modificaciones de la vida humana y las sociedades y culturas y, Colombia, siendo hoy uno de los países con

Análisis del proceso metodológico en programas de educación superior en modalidad virtual. Paula Andrea Díaz Guillen, Yamilhet Andrade Arango, Ana María Hincapié Zuleta y Adriana Patricia Uribe. 
mayor tradición en procesos de formación impartidos en modalidad presencial, presenta un gran avance con el incremento de programas académicos soportados en las innovaciones tecnológicas y lineamientos tecno-pedagógicos definidos para orientar los procesos desde el ciberespacio.

\section{Conclusiones}

La mayoría de las instituciones de educación superior en Colombia utilizan las plataformas educativas como punto de encuentro de los diferentes actores del proceso, además, aprovechan el ciberespacio como escenario principal de enseñanza-aprendizaje. Esto significa que, en su mayoría, estas instituciones implementan modelos educativos asincrónicos a partir de diseños de entornos virtuales basados en material de estudio, estrategias de evaluación y diferentes mediaciones educativas necesarias para apoyar los procesos académicos, comunicacionales y administrativos a lo largo del desarrollo de los programas de pregrado y posgrado.

La investigación permitió identificar aspectos metodológicos particulares de esta modalidad de estudio, tales como: (1) existencia de modelos pedagógicos que guían la planeación desde lo metodológico hasta la gestión de lo académico; (2) diseño de programas que privilegian la autoformación y la auto-regulación del estudiante, así como el seguimiento permanente de los docentes del proceso de enseñanza-aprendizaje; (3) aplicación de la evaluación formativa a lo largo del proceso, que permite ir evaluando, retroalimentando y fortaleciendo el mismo, con el fin de alcanzar las metas, objetivos o competencias propuestas; (4) prevalencia de la evaluación a través de cuestionarios y otras actividades en línea; y (5) variación de la relación del crédito para la organización de las actividades académicas, pero teniendo como centro del proceso al estudiante y su interacción con lo propuesto en el aula virtual.

Si bien las Instituciones de Educación Superior en Colombia cuentan con plataformas educativas que apoyan todo el proceso de enseñanza-aprendizaje (24/7), estas instituciones garantizan que se cumplan estándares de calidad en relación a los tiempos con base en las propuestas del número de créditos y en la relación establecida de los mismos (interacción académica con los docentes y trabajo independiente), lo cual varía de acuerdo con el nivel de formación de pregrados y posgrados.

De los resultados se destaca que las Instituciones de Educación Superior están comprometidas con las condiciones sociales y contextuales de las regiones y del país, por lo que han desarrollado la capacidad para entregar respuestas teniendo en cuentas las necesidades del medio y prácticas modernas que potencian la formación integral de los sujetos y la utilización de las TIC.

En síntesis, la sociedad del conocimiento ha sido clave para que la educación virtual haya ganado protagonismo en las Instituciones de Educación Superior. Se constituye dicha sociedad como fuente de desarrollo y progreso, y solo se puede concebir como el resultado de los procesos de globalización y de las innovaciones tecnológicas en comunicaciones que favorecen modalidades educativas que, en la actualidad, resultan

Análisis del proceso metodológico en programas de educación superior en modalidad virtual. Paula Andrea Díaz Guillen, Yamilhet Andrade Arango, Ana María Hincapié Zuleta y Adriana Patricia Uribe. 
estratégicas, esto es: sin fronteras, con multiplicidad de alianzas, con estudiantes y docentes ubicados en el mundo, estructuras flexibles de acceso, amplia variedad de ofertas y modelos pedagógicos nuevos que direccionen procesos diferentes a los lineales de aprendizaje soportado en las TIC.

Hoy día, se habla con propiedad del ciberespacio como lugar donde se aprende de manera individual y con los otros, donde se interactúa con el contenido y las actividades y los diferentes actores que participan del proceso educativo. Esto significa que, actualmente, el ser humano aprovecha las plataformas, los recursos de transmisión en línea y consulta interactiva, las bibliotecas, las bases de datos o la web en general de información, como también el conocimiento de otras personas. Se permite el lenguaje digital para representar, simular y recrear didácticamente la realidad sin limitaciones espacio-temporales.

Adicionalmente, hoy día, se reconoce plenamente la existencia de una sociedad que cada vez más, en el centro de sus desarrollos, ubica las TIC. Esto demuestra que es un hecho que la cultura de las sociedades se ve influenciada por las culturas digitales, asunto que está modificando las formas de transmitir y construir conocimiento. La ciencia y la tecnología están tamizando en este país por la presencia de ofertas de educación en especial desde las universidades, que, con criterio y sostenibilidad, transforman los formas de enseñar y de evaluar, con el objetivo de ampliar cobertura y facilitar el acceso a más personas.

Es necesario fortalecer las redes de trabajo académicas porque la educación virtual, en colaboración interinstitucional, favorecerá el crecimiento de la oferta educativa y el desarrollo del capital humano. Es relevante trabajar en elementos no solo que hagan posible esta modalidad, sino la misma calidad con base en las regulaciones, estándares y procedimientos relacionados con su actividad y la experiencia misma de las instituciones. Se deben definir procesos periódicos de evaluación del impacto, uso y relevancia de las herramientas y metodologías, casi de forma paralela a la implementación que garantice el crecimiento a escala de programas e incremento de estudiantes con modelos sostenibles y de calidad.

Presentación del artículo: 24 de octubre de 2020

Fecha de aprobación: 14 de diciembre de 2020

Fecha de publicación: 08 de enero de 2021

Díaz-Guillen, P.A., Andrade Arango, Y., Hincapié Zuleta, A.M., y Uribe, A.P. (2021). Análisis del proceso metodológico en programas de educación superior en modalidad virtual. RED. Revista Educación a Distancia, 21(65). https://doi.org/10.6018/red.450711

Análisis del proceso metodológico en programas de educación superior en modalidad virtual. Paula Andrea Díaz Guillen, Yamilhet Andrade Arango, Ana María Hincapié Zuleta y Adriana Patricia Uribe. 


\section{Financiación}

El presente texto nace en el marco del proyecto "Evaluación del proceso metodológico del sistema de educación a distancia de los programas de pregrado y posgrado de la Universidad de Manizales ofrecidos en metodología virtual frente a las categorías de calidad de la formación y la inclusión digital" (Resolución 42 septiembre 13 de 2017 financiado mediante convocatoria interna de investigación Universidad de Manizales).

\section{Referencias}

Abad, G. L., \& Orón, L. C. (2015). Espacio y tiempo en el siglo XXI: velocidad, instantaneidad y su repercusión en la comunicación humana. Revista ComHumanitas, 6(1), 219-233.

Alonso González, M. (2019). Fake News: desinformación en la era de la sociedad de la información. Ámbitos. Revista Internacional de Comunicación, 45, 29-52.

Amozurrutia, J. A., \& Moreno, M. M. (2007). Cibercultura e iniciación en la investigación (Vol. 11). UNAM.

Arango, Y., Díaz, P., \& Zuleta, A. (2018). La inclusión digital en los sistemas de educación superior en la modalidad virtual. En Orozco, E., Rodríguez, J., Gómez, C., \& Barrietos, A. (Eds.), Cultura digital y las nuevas políticas educativas (pp. 1526). Gedisa.

Areth-Estévez, J., Castro-Martínez, J., \& Rodríguez Granobles, H. (2015). La educación virtual en Colombia: exposición de modelos de deserción. Apertura, 7(1), $1-10$.

Basantes, A. V., Naranjo, M. E., \& Ojeda, V. (2018). Metodología PACIE en la Educación Virtual: una experiencia en la Universidad Técnica del Norte. Formación Universitaria, 11(2), 35-44. http://dx.doi.org/10.4067/S071850062018000200035

Berrío, C., Marín, P., Ferreira, E., \& Chagas, E. (2017). Desafíos de la Inclusión Digital: antecedentes, problemáticas y medición de la Brecha Digital de Género. Psicología, Conocimiento y Sociedad, 7(2), 121-151. http://dx.doi.org/10.26864/pcs.v7.n2.8.

Bonavitta, P. (2018). Las brechas digitales de género en la era digital de la información. En Casarin, M. (Ed), En torno a las ideas de Manuel Castells: discusiones en la era de la información (pp. 17-31). Cea Universidad Nacional de Córdoba.

Bradbrook, G., Alvi, I., Fisher, J., Lloyd, H., Moore, R., Thompson, V., Brake, D., Helsper, E., \& Livingstone, S. (2008). Meeting their potential: the role of education and technology in overcoming disadvantage and disaffection in young people. Becta.

Buzato, M. E. K. (2008). Inclusión digital como invención de lo cotidiano. Revista Brasileña de Educación, 13(38), 325-342. http://dx.doi.org/10.1590/S141324782008000200010

Cabrera, J. (2004). Navigators and castaways in cyberspace: psychosocial experience and cultural practices in school children's appropriation of the Internet. In: M.

Análisis del proceso metodológico en programas de educación superior en modalidad virtual. Paula Andrea Díaz Guillen, Yamilhet Andrade Arango, Ana María Hincapié Zuleta y Adriana Patricia Uribe. 
Bonilla; \& G. Cliché (Eds.), Internet and Society in Latin America and the Caribbean (pp. 21-86). Southbound / IDRC Books.

Cantón, I. (2000). Evaluación, cambio y calidad en las organizaciones educativas. Fundec.

Carreras, C. (2008). Evaluación TIC en el patrimonio cultural: metodologías y estudios de caso (Vol. 5). Editorial UOC.

Castells, M. (1999). La cultura de la virtualidad real: la integración de la comunicación electrónica, el fin de la audiencia de masas y el desarrollo de las redes interactivas. La era de la información, 1, 359-408.

Castells, M. (2006). La sociedad red: una visión global. Alianza.

Castro-Rodríguez, M., Marín-Suelves, D., \& Sáiz, H. (2019). Competencia digital e inclusión educativa. Visiones de profesorado, alumnado y familias. Revista De Educación a Distancia (RED), 19(61), 1-37. https://doi.org/10.6018/red/61/06

Catalán, J. H. T. (2018). Continuidades y desafíos de la Educación a Distancia: un estudio de caso. Revista electrónica de Investigación e Innovación educativa REIIE, 3(2), 39-58.

Centro de Educación a distancia Universidad de Manizales - CEDUM. (2019). Plan de Desarrollo. Universidad de Manizales.

CEPAL, N. (2018). Informe de la Sexta Conferencia Ministerial sobre la Sociedad de la Información de América Latina y el Caribe. https://repositorio.cepal.org/bitstream/handle/11362/44296/1/S1800305_es.pdf

Comisión Económica para América Latina y el Caribe - CEPAL (2003). Los caminos hacia una sociedad de la información en América Latina y el Caribe. Conferencia Ministerial Regional Preparatoria de América Latina y el Caribe para la Cumbre Mundial sobre la Sociedad de la Información.

Díaz, M. (2005). Cambio de paradigma metodológico en la Educación Superior Exigencias que conlleva (Cátedra de Universidad). Cuadernos de integración europea \# 2. http://repositori.uji.es/xmlui/bitstream/handle/10234/187445/cambio_Miguel_CIE 2005.pdf?sequence $=4$

Dussel, I. (2014). Programas educativos de inclusión digital. Versión. Estudios de Comunicación $\quad y \quad$ Política, 34, 39-56. https://versionojs.xoc.uam.mx/index.php/version/article/view/672/668

Escudero-Nahón, E. (2017). Aportaciones al proceso horizontal de transversalización de la Educación a Distancia en las instituciones de educación superior. Revista de la educación superior, 46(182), 57-69. https://doi.org/10.1016/j.resu.2017.02.003

Espinosa-Izquierdo, J., Peña-Hojas, D., Astudillo-Calderón, J., \& Coronel-Escobar, C. (2017). Multimedia educativa como recurso didáctico y su uso en el aula. Revista Científica $\quad$ Sinapsis, 1(10), $1-10$. https://doi.org/10.37117/s.v1i10.108

Estévez, J. A., Castro-Martínez, J., \& Granobles, H. R. (2015). La educación virtual en Colombia: exposición de modelos de deserción. Apertura, 7(1), 1-10. https://www.redalyc.org/pdf/688/68838021007.pdf

Fainholc, B. (2004). La calidad en la educación a distancia continúa siendo un tema muy complejo. Revista De Educación a Distancia (RED), 12, 27. https://revistas.um.es/red/article/view/25311

Análisis del proceso metodológico en programas de educación superior en modalidad virtual. Paula Andrea Díaz Guillen, Yamilhet Andrade Arango, Ana María Hincapié Zuleta y Adriana Patricia Uribe. 
Flórez-Romero, M., Aguilar-Barreto, A. J., Hernández Peña, Y. K., Salazar Torres, J. P., Pinillos-Villamizar, J. A., \& Pérez-Fuentes, C. A. (2017). Sociedad del conocimiento, las TIC y su influencia en la educación. Espacios, 38(35), 39.

Galindo, L. (2015). El aprendizaje colaborativo en ambientes virtuales. Cenid AC. Galperín, H. (2017). Sociedad digital: brechas y retos para la inclusión digital en América Latina y el Caribe. http://disde.minedu.gob.pe/handle/MINEDU/5803

García-Aretio, L. (2014). Bases, mediaciones y futuro de la educación a distancia en la sociedad digital. Edición Síntesis.

Garrison, D. R. (1989). Understanding distance education. Routledge.

González, A. M. (2018). La innovación educativa en educación superior: la modalidad blended learning. http://ridaa.unq.edu.ar/bitstream/handle/20.500.11807/786/TFI_2012_gonzalez_ 011.pdf? sequence $=1 \&$ isAllowed $=$

González-González, J., Gold-Morgan, M., Santamaría-Ambriz, R., Yáñez-Ordónez, O., \& Masjuán del Pino, M. (2011). Análisis estructural integrativo de Organización Universitaria: el modelo" $V^{\prime \prime}$ de evaluación-planeación como instrumento para el mejoramiento permanente de la educación superior. UDUAL, RIEV y UNAM.

Grande, M., Cañón, R., \& Cantón, I. (2016). Tecnologías de la información y la comunicación: evolución del concepto y características. IJERI: International Journal of Educational Research and Innovation, 6, 218-230. https://www.upo.es/revistas/index.php/IJERI/article/view/1703/1559

Gros-Salvat, B. (2011). Evolución y retos de la educación virtual. Editorial UOC.

Juca-Maldonado, F. X. (2016). La educación a distancia, una necesidad para la formación de los profesionales. Revista Universidad y Sociedad, 8(1), 106-111. http://scielo.sld.cu/scielo.php?script=sci_arttext\&pid=S221836202016000100016

Melo, D., \& Díaz, P. (2018). El aprendizaje afectivo y la gamificación en escenarios de educación virtual. Información Tecnológica, 29(3), $237-$ 248. http://dx.doi.org/10.4067/S0718-07642018000300237

Melo, D., Díaz, P., Vega, O., \& Serna, C. (2018). Situación Digital para Instituciones de Educación Superior: Modelo y Herramienta. Información Tecnológica, 29(6), 163-174. http://dx.doi.org/10.4067/S0718-07642018000600163

Ministerio de Educación Nacional, República de Colombia (2015). Decreto número 1075. Por medio del cual se expide el Decreto Único Reglamentario del Sector Educación. https://www.mineducacion.gov.co/1759/w3-article-351080.html

Ministerio de Educación Nacional, República de Colombia. (2009). Educación virtual o educación en línea: ¿Qué es la educación virtual? https://www.mineducacion.gov.co/1621/article-196492.html

Ministerio de Educación Nacional, República de Colombia. (2010). Lineamientos para la educación virtual en la educación superior. República de Colombia

Núñez, E., Miguelina, I., \& Ravina, R. (2019). El impacto de la utilización de la modalidad B-Learning en la educación superior. Alteridad, 14(1), 2639. https://doi.org/10.17163/alt.v14n1.2019.02.

Análisis del proceso metodológico en programas de educación superior en modalidad virtual. Paula Andrea Díaz Guillen, Yamilhet Andrade Arango, Ana María Hincapié Zuleta y Adriana Patricia Uribe. 
Organización de las Naciones Unidas para la Educación, la Ciencias y la Cultura UNESCO. (2013). Enfoques estratégicos sobre TIC en educación América Latina y el Caribe. Oficina Regional de Educación para América Latina y el Caribe.

Organización de las Naciones Unidas para la Educación, la Ciencias y la Cultura UNESCO. (2017) Educación para los objetivos de Desarrollo Sostenible: objetivos de aprendizaje. Oficina Regional de Educación para América Latina y el Caribe.

Otero de Suárez, P., Pineda, C., Rees, P. (2016). Herramientas de comunicación y presentación de contenidos en pregrados virtuales colombianos. Magis, 8(17). https://doi.org/10.11144/Javeriana.m8-17.hcpc

Padilla-Beltrán, J. E. (2016) La educación virtual en Colombia la implementación de las TIC en la educación superior. Revista Academia y Virtualidad, 4(1), 6-21. https://doi.org/10.18359/ravi.2618

Parra, Y. (2009). Perspectivas sociológicas sobre la educación virtual a distancia en Venezuela. ¿Una solución o un nuevo problema social para el acceso a la educación superior? Omnia, 15(3), 150-168.

Pedraja, L. (2017). Desafíos para la gestión pública en la sociedad del conocimiento. Interciencia, 42(3), 145. http://www.redalyc.org/pdf/339/33950011001.pdf.

Peña, J. A. (2010). La concepción filosófica de lo virtual en la educación virtual. Revista Colombiana de Educación, 58, 118-139.

Peña-Seminario, M, V., \& Aponte-González, M.C. (2019) Internacionalización conectiva: el currículo en un mundo en red (Primera edición) Dirección de Publicaciones de la Universidad Católica de Guayaquil, 2018.

Pérez-Zúñiga, R., Mercado-Lozano, P., Martínez-García, M., Mena-Hernández, E., \& Partida-Ibarra, J. Á. (2018). La sociedad del conocimiento y la sociedad de la información como la piedra angular en la innovación tecnológica educativa. RIDE. Revista Iberoamericana para la Investigación y el Desarrollo Educativo, 8(16), 847-870.

Plan Nacional Decenal de Educación 2016-2026. (2018). El camino hacia la calidad y la equidad. República de Colombia.

Rama, C. (2014). La virtualización como el nuevo paradigma educativo en América Latina. En Meléndez, J., Montalvo, Y., Rama, C., \& Calcaño, E. (eds.), Informe Educación Virtual y a Distancia en Puerto Rico, (11-17). https://virtualeduca.org/documentos/observatorio/2014/informe-educacion-virtual$\mathrm{y}$-a-distancia-en-puerto-rico.pdf\#page $=11$

Ramírez-Montoya, M. S. (2015). Acceso abierto y su repercusión en la Sociedad del Conocimiento: Reflexiones de casos prácticos en Latinoamérica. Education in the Knowledge Society, 16(1), 103-118. https://www.redalyc.org/pdf/5355/535554757007.pdf

Ramos-Azcuy, J. F. (2018). La evaluación de la calidad de los programas de posgrado online desde un enfoque de ciencia, tecnología y sociedad. Revista Atlante: Cuadernos de Educación y Desarrollo, 93. https://www.eumed.net/rev/atlante/2018/03/calidad-programas-posgrado.html

Rengifo-Millán, M. (2015). La globalización de la sociedad del conocimiento y la transformación universitaria. Revista Latinoamericana de Ciencias Sociales, Niñez y Juventud, 13(2), 809-822.

Análisis del proceso metodológico en programas de educación superior en modalidad virtual. Paula Andrea Díaz Guillen, Yamilhet Andrade Arango, Ana María Hincapié Zuleta y Adriana Patricia Uribe. 
Rivoir, A. (2009). Innovación para la inclusión digital. El Plan Ceibal en Uruguay. Revista Mediaciones Sociales, 4, 299-328

Rodríguez, S.M. (2015). Redes de colaboración académica: una respuesta a los desafíos de la globalización y la sociedad del conocimiento. Sinéctica, 44, 1-18.

Roquet-García, G. (2006). Antecedentes históricos de la educación a distancia. Universidad Nacional Autónoma de México.

Salazar, E., \& Tobón, S. (2018). Análisis documental del proceso de formación docente acorde con la sociedad del conocimiento. Revista Espacios, 39(53), 17.

Salazar, R., Melo, A, Arboleda, N., \& Rama, C. (2013). Lineamientos conceptuales de la modalidad de educación a distancia: la educación superior a distancia y virtual en Colombia: nuevas realidades. ACESAD.

Sánchez, I. R. A. (2016). La Sociedad de la Información, Sociedad del Conocimiento y Sociedad del Aprendizaje. Referentes en torno a su formación. Bibliotecas. Anales de Investigación, 12(2), 235-243.

Sánchez-Torres, J. M., González-Zabala, M. P., \& Muñoz, M. P. S. (2012). La sociedad de la información: génesis, iniciativas, concepto y su relación con las TIC. Revista UIS Ingenierías, 11(1), 113-128.

Serrano, V. (2015). Tras el ensayo digital: una aproximación interdisciplinar a la sociedad de la información (Vol. 3). Ediciones Universidad Austral de Chile.

Shea, P., \& Bidjerano, T. (2009). Community of inquiry as a theoretical framework to foster "epistemic engagement" and "cognitive presence" in online education. Computers \& Education, 52(3), 543-553.

Silvio, J. (2009). Reflexiones sobre la calidad en la educación virtual. Organización de los Estados Americanos (OEA), Departamento de Asuntos Educativos.

Soto, A. P. G., \& Miró, J. D. F. (2016). Usabilidad y accesibilidad para un e-learning inclusivo. Revista de Educación Inclusiva, 2(1). 49-60.

Taylor, J. C. (1999). Distance Education. The Fifth Generation. Trabajo presentado a la IXX Conferencia Mundial del ICDE. Viena.

Tinoco-Giraldo, H., \& Giraldo, J. I. Z. (2019). Evaluación de la percepción del impacto de las prácticas académicas: una mirada desde los escenarios de aprendizaje. Lúmina, (20), 30-53.

Tovar-Gálvez, J. C., \& Cárdenas-Puyo, N. (2012). La importancia de la formación estratégica en la formación por competencias: evaluación de las estrategias de acción para la solución de problemas. Revista Electrónica de Investigación Educativa, 14(1), 122-135

\section{Anexo 1}

\section{Guía de Entrevista}

\section{Enseñanza-aprendizaje}

¿Cuáles es el referente o el modelo pedagógico que su institución desarrolla bajo la modalidad virtual?

¿Cómo se verifica su aplicación en el proceso de enseñanza-aprendizaje?

Análisis del proceso metodológico en programas de educación superior en modalidad virtual. Paula Andrea Díaz Guillen, Yamilhet Andrade Arango, Ana María Hincapié Zuleta y Adriana Patricia Uribe. 
¿Cómo define su institución las formas, los tiempos, los lugares y los momentos de evaluación de los estudiantes?

¿Cómo la institución da cuenta del tiempo de trabajo independiente del estudiante, los momentos de tutorías y los momentos de acompañamiento (ya sean presenciales, virtuales, sincrónicos o asincrónicos)?

¿Cómo se miden (ejemplo: ¿relación de créditos, horas de interacción, asignación docente, entrega de actividades, semanas, entre otros)?

¿Cuáles son las principales estrategias de enseñanza y los medios educativos requeridos por los programas?

¿Cuáles son las estrategias que tiene su institución para dar acompañamiento académico (bienestar universitario, psicología, asesoría académica)?

\section{Evaluación}

¿Qué estrategias de evaluación curricular utilizan en los programas que se orientan en la modalidad virtual de su Universidad?

\section{Perfil estudiante}

¿Qué programa presenta su institución como plan de alfabetización digital para los estudiantes que ingresan en la modalidad virtual?

¿Cómo al estudiante y su rol en el proceso de enseñanza-aprendizaje?

\section{Perfil docente/tutor}

¿Quién opera como vínculo personal-mediador entre el estudiante y las diferentes instancias administrativas, el proceso académico, el docente, los contenidos y los recursos tecnológicos?

¿Quién o quiénes son los encargados del diseño/construcción de material de estudio, la orientación, el seguimiento y evaluación del proceso de aprendizaje?

¿Cómo diseñan los programas, las formas de contacto e interacción entre los profesores (tutores, monitores) y los estudiantes para cada uno de los momentos de trabajo y evaluaciones?

¿En qué consiste el plan de desarrollo profesoral didáctico y de competencias digitales articulado a la modalidad?

¿Cómo la institución logra la articulación de los materiales y los recursos de estudio desarrollados y utilizados por los docentes con los lineamientos institucionales de propiedad intelectual?

\section{Créditos académicos}

¿Cómo establecen la relación del crédito de forma que el estudiante conozca de cuánto tiempo debe disponer para el adecuado desarrollo de cada actividad propuesta, de los momentos de acompañamiento sincrónico y el trabajo independiente?

¿Cómo vinculan los programas virtuales los estudiantes a los procesos de investigación?

\section{Investigación}

¿Cómo los programas determinan las necesidades bibliográficas, de equipos computacionales y aplicativos informáticos generales y especializados, y de conectividad requeridas para el desarrollo adecuado del currículo propuesto?

Análisis del proceso metodológico en programas de educación superior en modalidad virtual. Paula Andrea Díaz Guillen, Yamilhet Andrade Arango, Ana María Hincapié Zuleta y Adriana Patricia Uribe. 
¿Con qué plan o estrategia cuentan los programas como base de seguimiento del uso que profesores y estudiantes realizan de los medios educativos y de la infraestructura de la institución? 